\title{
To the theory of semi-linear equations in the plane
}

\author{
Vladimir Gutlyanskĭ̌, Olga Nesmelova, Vladimir Ryazanov
}

\section{Dedicated to the memory of Professor Bogdan Bojarski}

\begin{abstract}
In two dimensions, we present a new approach to the study of the semi-linear equations of the form $\operatorname{div}[A(z) \nabla u]=f(u)$, the diffusion term of which is the divergence uniform elliptic operator with measurable matrix functions $A(z)$, whereas its reaction term $f(u)$ is a continuous non-linear function. Assuming that $f(t) / t \rightarrow 0$ as $t \rightarrow \infty$, we establish a theorem on existence of weak $C(\bar{D}) \cap W_{\text {loc }}^{1,2}(D)$ solutions of the Dirichlet problem with arbitrary continuous boundary data in any bounded domains $D$ without degenerate boundary components. As consequences, we give applications to some concrete model semi-linear equations of mathematical physics, arising from modelling processes in anisotropic and inhomogeneous media. With a view to further development of the theory of boundary value problems for the semi-linear equations, we prove a theorem on the solvability of the Dirichlet problem for the Poisson equation in Jordan domains with arbitrary boundary data that are measurable with respect to the logarithmic capacity.
\end{abstract}

2010 MSC. Primary 30C62, 31A05, 31A20, 31A25, 31B25, 35J61 Secondary 30E25, 31C05, 34M50, 35Q15.

Key words and phrases. Semi-linear elliptic equations, quasilinear Poisson equations, anisotropic and inhomogeneous media, conformal and quasiconformal mappings.

\section{Introduction}

The study of linear and non-linear elliptic partial differential equations in two dimensions by the methods of complex analysis and quasiconformal mappings with concrete applications in nonlinear elasticity, gas flow, hydrodynamics and other sections of natural science has been initiated by M. A. Lavrentiev [54], C. B. Morrey [60], L. Bers [8], L. Bers and L. Nirenberg [9], I. N. Vekua [72], B. Bojarski [11], J. Serrin [68] and others, see the references therein. The history of such equations actually

Received 29.03.2019 
goes back as far as the celebrated works by d'Alembert on the CauchyRiemann systems in hydrodynamics, by Gauss on geometry of surfaces, by Lobaczewski on non-Euclidean geometry, and by the pioneer paper of E. Beltrami [7].

A rather comprehensive treatment of the present state of the theory is given in the excellent book of Astala, Iwaniec and Martin [4]. This book relates the most modern aspects and most recent developments in the theory of planar quasiconformal mappings and their application in conformal geometry, partial differential equations and nonlinear analysis. The book contains also an exhaustive bibliography on the topic. Among the variety of deep results on this topic we single out the fundamental Harmonic factorization theorem, see [4], Theorem 16.2.1, for the solutions to the non-linear uniformly elliptic divergence equations

$$
\operatorname{div} A(z, \nabla u)=0, \quad z \in D \subset \mathbb{C},
$$

and the corresponding regularity results. In particular, the factorization theorem claims that every solution $u \in W_{\text {loc }}^{1,2}(D)$ of the equation (1.1) can be expressed as $u(z)=h(f(z))$, where $f: D \rightarrow G$ is $K$-quasiconformal and $h$ is harmonic on $G$.

In series of our recent papers [33-40], we have proposed another application of the quasiconformal mappings theory to the the study of semilinear partial differential equations of the form

$$
\operatorname{div}[A(z) \nabla u(z)]=f(u), z \in D, D \subseteq \mathbb{C},
$$

the diffusion term of which is the divergence form elliptic operator $L(u)$, whereas its reaction term $f(u)$ is a non-linear function. Here the symmetric matrix function $A(z)=\left\{a_{i j}(z)\right\}$, $\operatorname{det} A(z)=1$, with measurable entries satisfies the uniform ellipticity condition

$$
\frac{1}{K}|\xi|^{2} \leq\langle A(z) \xi, \xi\rangle \leq K|\xi|^{2} \text { a.e. in } D, 1 \leq K<\infty,
$$

for every $\xi \in \mathbb{R}^{2}$. The set of all such matrix functions we denoted by $M_{K}^{2 \times 2}(D)$. In the cited papers we have studied the composition properties of $L(u)$ first for sufficiently smooth functions and then in the Sobolev spaces making use of the fundamental compositional theorems established in $[28,70]$. It was shown that by the chain rule for the function $u=U \circ \omega$ the following basic formula holds

$$
\operatorname{div}[A(z) \nabla(U(\omega(z)))]=J_{\omega}(z) \triangle U(\omega(z)),
$$

where $J_{\omega}(z)$ stands for the Jacobian of the quasiconformal mapping $\omega$, agreed with the matrix function $A(z)$. This formula, which is understood 
in the sense of distributions, takes place, in particular, if $U \in W_{\text {loc }}^{1,2}(G)$, $A \in M_{K}^{2 \times 2}(D)$ and $\omega: D \rightarrow G$ is a quasiconformal homeomorphism satisfying the Beltrami equation

$$
\omega_{\bar{z}}(z)=\mu(z) \omega_{z}(z) \text { a.e. in } D .
$$

Here the complex dilatation

$$
\mu(z)=\frac{1}{\operatorname{det}(I+A)}\left(a_{22}-a_{11}-2 i a_{12}\right),
$$

satisfies the uniform ellipticity condition

$$
|\mu(z)| \leq \frac{1+K}{1-K} .
$$

Vice versa, given measurable complex valued function $\mu$, satisfying (1.7), one can invert the algebraic system (1.6) to obtain

$$
A(z)=\left(\begin{array}{cc}
\frac{|1-\mu|^{2}}{1-|\mu|^{2}} & \frac{-2 \operatorname{Im} \mu}{1-|\mu|^{2}} \\
\frac{-2 \operatorname{Im} \mu}{1-|\mu|^{2}} & \frac{|1+\mu|^{2}}{1-|\mu|^{2}}
\end{array}\right) .
$$

The compositional property (1.4) for the operator $L(u)$ can be applied to the study of a wide range of problems arising in the contemporary analysis in the plane. For instance, (1.4) is useful for the study of such semi-linear partial differential equations in anisotropic case as the heat equation

$$
u_{t}-\operatorname{div}[A(z) \nabla u(z)]=f(z, u),
$$

(the same equation describes the brownian motion, diffusion models of population dynamics, and many other phenomena), the wave equation

$$
u_{t t}-\operatorname{div}[A(z) \nabla u(z)]=f(z, u)
$$

and the Schrödinger equation

$$
i u_{t}+\operatorname{div}[A(z) \nabla u(z)]=k|u|^{p} u,
$$

as well as their stationary counterparts. Note also a very interesting recent preprint [27], where the authors have developed a method for the study of spectral properties of the operators $L(u)$ with the Neumann boundary condition in (non)convex domains in the complex plane. The suggested method is based on the composition operators on Sobolev spaces with applications to the Poincare inequalities. 
The composition property (1.4) for the operator $L(u)$ implies that the study of the semi-linear equations of the form (1.2) is decomposed into the research of a proper quasi-linear Poisson's equation

$$
\triangle U(w)=J(w) f(U), \quad w \in G=\omega(D),
$$

where the weight $J(w)$ stands for the Jacobian of the inverse quasiconformal mapping $\omega^{-1}: G \rightarrow D$, and the study of the mapping $\omega(z)$ agreed with the matrix function $A(z)$. In other words, every weak solution $u(z)$ to the equation (1.2) in a domain $D$ is represented in the form $u(z)=U(\omega(z))$ where $\omega: D \rightarrow G$ stands for a quasiconformal mapping generated by the matrix function $A(z)$ and $U(w)$ is a weak solution to the quasilinear Poisson equation (1.12) in the domain $G=\omega(D)$, see [36], Theorem 4.1 .

On the one hand, this opens up new possibilities for the study of (1.12), because we can apply a wide range of effective methods both of the potential theory and genuinely nonlinear methods which did not belong to the world of classical harmonic analysis, see e.g. [16, 26, 30, 51, $52,64,68$ ], and the exhaustive bibliography therein. On the other hand, a comprehensively developed theory of quasiconformal mappings in the plane, see e.g. [1, 4, 12, 13, 42,55], and also [41, 43], allows us to study in detail both the regularity properties for solutions to the equations (1.2), (1.12) and the proper representation of such solutions. A rather comprehensive treatment of the present state of the theory concerning the semi-linear equations of the form (1.12) is given in the excellent books of M. Marcus and L. Véron [58] and L. Véron [73].

Note an important family of quasi-linear Poisson's equations that involves an absorption term such that $u f(u) \geq 0$. Such equations are of particular interest because, in particular in them we have two competing effects, observed in a number of applications: the diffusion expressed by the linear differential part and the absorption produced by the nonlinearity of the right hand side. Among the verity of model semi-linear equations in the plane recall also the Liouville-Bieberbach equation

$$
\triangle u=e^{u}
$$

investigated by Bieberbach in his celebrated work [10] related to the study of automorphic functions in the plane.

The Liouville-Bieberbach semi-linear equation is one of the principle model equations in the theory of non-linear partial differential equations and their applications, see e.g. [58] and the references therein. Note that the equation appears also as a model one in problems of differential geometry in relation with existence of surfaces of negative Gaussian curvature [72] and in studying the equilibrium of a charged gas. 
In order to illustrate our approach to the study of the equation (1.2), we complete the introduction with several non-trivial model examples. The corresponding proofs together with other examples have been given in [36] and [38].

$\mathrm{n} 1$. Assume that the reaction term in (1.2) is non-negative. Since $u=$ $U(\omega(z))$ and $U$ satisfies in $G=\omega(D)$ the equation (1.12) we see, having in mind that the Jacobian $J(w)$ of the quasiconformal mapping $\omega^{-1}(w)$ is non-negative almost everywhere in $G$, that $U$ is a subharmonic function in $G$. Thus we arrive at the following generalization of the mentioned above Harmonic factorization theorem: Every solution $u \in W_{\mathrm{loc}}^{1,2}(D)$ of the semi-linear equation (1.2) with $f(u) \geq 0$ can be expressed as $u(z)=$ $U(\omega(z))$, where $\omega: D \rightarrow G$ is a $K$-quasiconformal mapping agreed with the matrix function $A(z)$ and $U$ is subharmonic in $G$.

$\mathrm{n} 2$. Let us consider the divergence form of the model LiouvilleBieberbach semi-linear equation

$$
\operatorname{div}[A(z) \nabla u]=e^{u}, \quad z=x+i y,
$$

in the unit disk $\mathbb{D}=\{z:|z|<1\}$. If the matrix function $A(z)$ has the following entries

$$
\begin{gathered}
a_{11}=3-2 \frac{x^{2}-y^{2}-2 x y}{\left(x^{2}+y^{2}\right)}, a_{12}=-2 \frac{x^{2}-y^{2}+2 x y}{\left(x^{2}+y^{2}\right)}, \\
a_{22}=3+2 \frac{x^{2}-y^{2}-2 x y}{\left(x^{2}+y^{2}\right)},
\end{gathered}
$$

it is easy to verify that the function

$$
u(x, y)=-2 \log \left(1-x^{2}-y^{2}\right)+\log 8
$$

realizes the blow-up solution to the equation (1.14) in the disk $\mathbb{D}$. In this case the matrix function $A(z)$ generates the well-known logarithmic spiral quasiconformal mapping

$$
\omega(z)=z e^{2 i \log |z|}
$$

which plays important role in the study of different problems of contemporary analysis, see, e.g., $[13, \S 13.2],[22,32]$. This function $\omega$ maps the unit disk $\mathbb{D}$ onto itself and transforms radial lines into spirals, infinitely winding about the origin. Since the mapping $\omega$ is volume preserving, the problem (1.14) is reduced to the well-known solvability result, see [10] and also [58], Theorem 5.3.7, for the Liouville-Bieberbach equation (1.13). 
n 3. Let $\mathbb{C}$ be the complex plane and let

$$
A(z)=\left(\begin{array}{cc}
1 & \mp \frac{2 \nu(x)}{\sqrt{1-\nu^{2}(x)}} \\
\mp \frac{2 \nu(x)}{\sqrt{1-\nu^{2}(x)}} & \frac{1+3 \nu^{2}(x)}{1-\nu^{2}(x)}
\end{array}\right)
$$

where $\nu(x), x \in \mathbb{R}$, stands for an arbitrary measurable real-valued function, such that $|\nu(x)| \leq k<1$. Then the semi-linear equation

$$
\operatorname{div}[A(z) \nabla u]=u^{q}, \quad 0<q<1, \quad z \in \mathbb{C},
$$

has in the complex plane the following solution with the "dead zone":

$$
u(x, y)=\left\{\begin{array}{cc}
\gamma\left(y \pm \int_{0}^{x} \frac{2 \nu(t)}{\sqrt{1-\nu^{2}(t)}} d t\right)^{\frac{2}{1-q}}, & \text { if } y>\varphi(x), x \in \mathbb{R} \\
0 & \text { if } x \leq \varphi(x) .
\end{array}\right.
$$

Here

$$
\gamma=\left(\frac{(1-q)^{2}}{2(1+q)}\right)^{\frac{1}{1-q}}
$$

and

$$
y=\varphi(x)= \pm \int_{0}^{x} \frac{2 \nu(t) d t}{\sqrt{1-\nu^{2}(t)}}, \infty<x<+\infty,
$$

stands for the corresponding free boundary parametrization.

In this paper, for the sake of completeness, we will collect some basic facts from our recent research concerning semi-linear partial differential equations in the plane and give a number of new results on the topic. The paper is organized as follows. In Section 2 we give basic facts from the potential theory. In Sections 3 and 4 one can find existence theorems for the quasilinear Poisson equation (1.12) as well as for the corresponding semi-linear equation (1.2) without boundary conditions. We study the solvability of the Dirichlet problem with arbitrary continuous boundary data for the quasilinear Poisson equations (1.12) in Section 5. Section 6 is devoted to the solvability of the Dirichlet problem with continuous boundary data for semi-linear equation (1.2) and it also contains some applications. In the rest sections we discuss the boundary value problem for the Poisson equations with boundary data that are measurable with respect to the logarithmic capacity. 


\section{Basic facts from the potential theory}

For the sake of completeness we repeat the fundamental results concerning the potential theory in the plane, given in [35,39], and strengthen some of them.

From now on, $\mathbb{D}$ denotes the unit disk $\{z \in \mathbb{C}:|z|<1\}$ in the complex plane $\mathbb{C}, \mathbb{D}_{R}\left(z_{0}\right):=\left\{z \in \mathbb{C}:\left|z-z_{0}\right|<R\right\}$ for $z_{0} \in \mathbb{C}$ and $R \in(0, \infty)$, $\mathbb{D}_{R}:=\mathbb{D}_{R}(0)$.

For $z$ and $w \in \mathbb{D}$ with $z \neq w$, set

$$
G(z, w):=\log \left|\frac{1-z \bar{w}}{z-w}\right| \text { and } P\left(z, e^{i t}\right):=\frac{1-|z|^{2}}{\left|1-z e^{-i t}\right|^{2}}
$$

be the Green function and Poisson kernel in $\mathbb{D}$. If $\varphi \in C(\partial \mathbb{D})$ and $g \in C(\overline{\mathbb{D}})$, then a solution to the Poisson equation

$$
\triangle f(z)=g(z)
$$

satisfying the boundary condition $\left.f\right|_{\partial \mathbb{D}}=\varphi$ is given by the formula

$$
f(z)=\mathcal{P}_{\varphi}(z)-\mathcal{G}_{g}(z)
$$

where

$\mathcal{P}_{\varphi}(z)=\frac{1}{2 \pi} \int_{0}^{2 \pi} P\left(z, e^{i t}\right) \varphi\left(e^{-i t}\right) d t, \quad \mathcal{G}_{g}(z)=\int_{\mathbb{D}} G(z, w) g(w) d m(w)$,

see e.g. [45], p. 118-120. Here $m(w)$ denotes the Lebesgue measure in $\mathbb{C}$.

Next, we give the representation of solutions to the Poisson equation in the form of the Newtonian (normalized antilogarithmic) potential.

Given a finite Borel measure $\nu$ on $\mathbb{C}$ with compact support, its potential is the function $p_{\nu}: \mathbb{C} \rightarrow[-\infty, \infty)$ defined by

$$
p_{\nu}(z)=\int_{\mathbb{C}} \ln |z-w| d \nu(w)
$$

see [64], point 3.1.1.

Remark 1. Note that the function $p_{\nu}$ is subharmonic by Theorem 3.1.2 and, consequently, it is locally integrable on $\mathbb{C}$ by Theorem 2.5.1 in [64]. Moreover, $p_{\nu}$ is harmonic outside of the support of $\nu$.

This definition can be extended to finite charges $\nu$ with compact support (named also signed measures), i.e., to real valued sigma-additive 
functions on Borel sets in $\mathbb{C}$, because of $\nu=\nu^{+}-\nu^{-}$where $\nu^{+}$and $\nu^{-}$are Borel measures by the well-known Jordan decomposition, see e.g. Theorem 0.1 in [52].

The key fact is the following statement, see e.g. Theorem 3.7.4 in [64].

Proposition 1. Let $\nu$ be a finite charge with compact support in $\mathbb{C}$. Then

$$
\triangle p_{\nu}=2 \pi \cdot \nu
$$

in the distributional sense, i.e.,

$$
\int_{\mathbb{C}} p_{\nu}(z) \triangle \psi(z) d m(z)=2 \pi \int_{\mathbb{C}} \psi(z) d \nu(z) \quad \forall \psi \in C_{0}^{\infty}(\mathbb{C}) .
$$

Here as usual $C_{0}^{\infty}(\mathbb{C})$ denotes the class of all infinitely differentiable functions $\psi: \mathbb{C} \rightarrow \mathbb{R}$ with compact support in $\mathbb{C}, \triangle=\frac{\partial^{2}}{\partial x^{2}}+\frac{\partial^{2}}{\partial y^{2}}$ is the Laplace operator and $d m(z)$ corresponds to the Lebesgue measure in $\mathbb{C}$.

Corollary 1. In particular, if for every Borel set $B$ in $\mathbb{C}$

$$
\nu(B):=\int_{B} g(z) d m(z)
$$

where $g: \mathbb{C} \rightarrow \mathbb{R}$ is an integrable function with compact support, then

$$
\triangle N_{g}=g
$$

where

$$
N_{g}(z):=\frac{1}{2 \pi} \int_{\mathbb{C}} \ln |z-w| g(w) d m(w),
$$

in the distributional sense, i.e.,

$$
\int_{\mathbb{C}} N_{g}(z) \triangle \psi(z) d m(z)=\int_{\mathbb{C}} \psi(z) g(z) d m(z) \quad \forall \psi \in C_{0}^{\infty}(\mathbb{C}) .
$$

Here the function $g$ is called a density of the charge $\nu$ and the function $N_{g}$ is said to be the Newtonian potential of $g$.

The next statement on continuity in the mean of functions $\psi: \mathbb{C} \rightarrow \mathbb{R}$ in $L^{q}(\mathbb{C}), q \in[1, \infty)$, with respect to shifts is useful for the study of the Newtonian potential, see e.g. Theorem 1.4.3 in [69], cf. also Theorem III(11.2) in [67]. The one-dimensional analog of the statement can be found also in [65], Theorem 9.5. 
Lemma 1. Let $\psi \in L^{q}(\mathbb{C}), q \in[1, \infty)$, have a compact support. Then

$$
\lim _{\Delta z \rightarrow 0} \int_{\mathbb{C}}|\psi(z+\Delta z)-\psi(z)|^{q} d m(z)=0 \text {. }
$$

Recall that a shift of a set $E \subset \mathbb{C}$ by a vector $\Delta z \in \mathbb{C}$ is the set

$$
E+\Delta z:=\{\xi \in \mathbb{C}: \xi=z+\Delta z, z \in E\} .
$$

We prefer to give a direct proof of this important statement that may be of independent interest. The proof is based on arguments by contradiction and the absolute continuity of indefinite integrals.

Proof. Let us assume that there is a sequence $\Delta z_{n} \in \mathbb{C}, n=1,2, \ldots$, such that $\Delta z_{n} \rightarrow 0$ as $n \rightarrow \infty$ and, for some $\delta>0$ and $\psi_{n}(z):=\psi\left(z+\Delta z_{n}\right)$, $n=1,2, \ldots$,

$$
I_{n}:=\left[\int_{\mathbb{C}}\left|\psi_{n}(z)-\psi(z)\right|^{q} d m(z)\right]^{\frac{1}{q}} \geq \delta \quad \forall n=1,2, \ldots .
$$

Denote by $K$ the closed disk in $\mathbb{C}$ centered at 0 with the minimal radius $R$ that contains the support of $\psi$. By the Luzin theorem, see e.g. Theorem 2.3.5 in [20], for every prescribed $\varepsilon>0$, there is a compact set $C \subset K$ such that $\left.g\right|_{C}$ is continuous and $m(K \backslash C)<\varepsilon$. With no loss of generality, we may assume that $C \subset K_{*}$ where $K_{*}$ is a closed disk in $\mathbb{C}$ centered at 0 with a radius $r \in(0, R)$ and, moreover, that $C_{n} \subset K$, where $C_{n}:=C-\Delta z_{n}$, for all $n=1,2, \ldots$. Note that $m\left(C_{n}\right)=m(C)$ and then $m\left(K \backslash C_{n}\right)<\varepsilon$ and, consequently, $m\left(K \backslash C_{n}^{*}\right)<2 \varepsilon$, where $C_{n}^{*}:=C \cap C_{n}$, because $K \backslash C_{n}^{*}=\left(K \backslash C_{n}\right) \cup(K \backslash C)$.

Next, setting $K_{n}=K-\Delta z_{n}$, we see that $K \cup K_{n}=C_{n}^{*} \cup\left(K \backslash C_{n}^{*}\right) \cup$ $\left(K_{n} \backslash C_{n}^{*}\right)$ and that $K_{n} \backslash C_{n}^{*}+\Delta z_{n}=K \backslash C_{n}^{*}$. Hence by the triangle inequality for the norm in $L^{p}$ the following estimate holds

$I_{n} \leq 4 \cdot\left[\int_{K \backslash C_{n}^{*}}|\psi(z)|^{q} d m(z)\right]^{\frac{1}{q}}+\left[\int_{C_{n}^{*}}\left|\psi_{n}(z)-\psi(z)\right|^{q} d m(z)\right]^{\frac{1}{q}} \forall n=1,2, \ldots$

By construction the both terms from the right hand side can be made to be arbitrarily small, the first one for small enough $\varepsilon$ because of absolute continuity of indefinite integrals and the second one for all large enough $n$ after the choice of the set $C$. Thus, the assumption (2.13) is disproved. 
Let $(X, d)$ and $\left(X^{\prime}, d^{\prime}\right)$ be metric spaces with distances $d$ and $d^{\prime}$, respectively. A family $\mathfrak{F}$ of mappings $f: X \rightarrow X^{\prime}$ is called equicontinuous at a point $x_{0} \in X$ if, for every $\varepsilon>0$, there is $\delta>0$ such that $d^{\prime}\left(f(x), f\left(x_{0}\right)\right)<\varepsilon$ for all $f \in \mathfrak{F}$ and $x \in X$ with $d\left(x, x_{0}\right)<\delta$. The family $\mathfrak{F}$ is said to be equicontinuous if $\mathfrak{F}$ is equicontinuous at every point $x_{0} \in X$.

Lemma 2. Let $g: \mathbb{C} \rightarrow \mathbb{R}$ be in $L^{p}(\mathbb{C})$ for $p>1$ with compact support. Then $N_{g}$ is continuous. A collection $\left\{N_{g}\right\}$ is equicontinuous if the collection $\{g\}$ is bounded by the norm in $L^{p}(\mathbb{C})$ with supports in a fixed disk K. Moreover, under the latter hypothesis, on each compact set $S$ in $\mathbb{C}$

$$
\left\|N_{g}\right\|_{C} \leq M \cdot\|g\|_{p}
$$

where $M$ is a constant depending in general on $S$ but not on $g$.

Proof. By the Hölder inequality with $\frac{1}{q}+\frac{1}{p}=1$ we have that

$$
\begin{gathered}
\left|N_{g}(z)-N_{g}(\zeta)\right| \leq \frac{\|g\|_{p}}{2 \pi} \cdot\left[\int_{K}|\ln | z-w|-\ln | \zeta-w||^{q} d m(w)\right]^{\frac{1}{q}} \\
=\frac{\|g\|_{p}}{2 \pi} \cdot\left[\int_{\mathbb{C}}\left|\psi_{\zeta}(\xi+\Delta z)-\psi_{\zeta}(\xi)\right|^{q} d m(\xi)\right]^{\frac{1}{q}}
\end{gathered}
$$

where $\xi=\zeta-w, \Delta z=z-\zeta, \psi_{\zeta}(\xi):=\chi_{K+\zeta}(\xi) \ln |\xi|$. Thus, the first two conclusions follow by Lemma 1 because of the function $\ln |\xi|$ belongs to the class $L_{\text {loc }}^{q}(\mathbb{C})$ for all $q \in[1, \infty)$.

The third conclusion similarly follows through the direct estimate

$$
\begin{aligned}
\left|N_{g}(\zeta)\right| & \leq \frac{\|g\|_{p}}{2 \pi} \cdot\left[\int_{K}|\ln | \zeta-\left.w\right|^{q} d m(w)\right]^{\frac{1}{q}} \\
& =\frac{\|g\|_{p}}{2 \pi} \cdot\left[\int_{\mathbb{C}}\left|\psi_{\zeta}(\xi)\right|^{q} d m(\xi)\right]^{\frac{1}{q}}
\end{aligned}
$$

because the latter integral is continuous in $\zeta \in \mathbb{C}$. Indeed, by the triangle inequality for the norm in $L^{q}(\mathbb{C})$ we see that

$$
\left|\left\|\psi_{\zeta}\right\|_{q}-\left\|\psi_{\zeta_{*}}\right\|_{q}\right| \leq\left\|\psi_{\zeta}-\psi_{\zeta_{*}}\right\|_{q}=\left\{\left.\int_{\Delta}|\ln | \xi\right|^{q} d m(\xi)\right\}^{\frac{1}{q}}
$$


where $\Delta$ denotes the symmetric difference of the disks $K+\zeta$ and $K+\zeta_{*}$. Thus, the statement follows from the absolute continuity of the indefinite integral.

The corresponding statement on the continuity of integrals of potential type in higher dimensions can be found in [69], Theorem 1.6.1.

Proposition 2. There exist functions $g \in L^{1}(\mathbb{C})$ with compact support whose potentials $N_{g}$ are not continuous, furthermore, $N_{g} \notin L_{\mathrm{loc}}^{\infty}$.

Proof. Indeed, let us consider the function

$$
\omega(t):=\frac{1}{t^{2}(1-\ln t)^{\alpha}}, t \in(0,1], \alpha \in(1,2), \quad \omega(0):=\infty
$$

and, correspondingly,

$$
g(z):=\omega(|z|), z \in \overline{\mathbb{D}}, \quad g(z):=0, z \in \mathbb{C} \backslash \overline{\mathbb{D}}
$$

Then, setting $\Omega(t)=t \cdot \omega(t)$, we see, firstly, that

$$
\int_{\overline{\mathbb{D}}}|g(w)| d m(w)=2 \pi \lim _{\rho \rightarrow+0} \int_{\rho}^{1} \Omega(t) d t=2 \pi \lim _{\rho \rightarrow+0} \int_{\rho}^{1} \frac{d \ln t}{(1-\ln t)^{\alpha}}=\frac{2 \pi}{\alpha-1}
$$

and, secondly, that the Newtonian potential $N_{g}$ at the origin is equal to

$$
\begin{gathered}
\lim _{\rho \rightarrow+0} \int_{\rho}^{1} \Omega(t) \ln t d t=\lim _{\rho \rightarrow+0}\left\{\left[\ln \frac{1}{t} \int_{t}^{1} \Omega(\tau) d \tau\right]_{\rho}^{1}+\int_{\rho}^{1}\left(\frac{1}{t} \int_{t}^{1} \Omega(\tau) d \tau\right) d t\right\} \\
=\frac{1}{\alpha-1} \cdot \lim _{\rho \rightarrow+0}\left(\left[\frac{\ln t}{(1-\ln t)^{\alpha-1}}\right]_{\rho}^{1}-\int_{\rho}^{1} \frac{d t}{t(1-\ln t)^{\alpha-1}}\right) \\
=\frac{1}{\alpha-1} \cdot \lim _{\rho \rightarrow+0}\left[\frac{1}{(1-\ln t)^{\alpha-1}}+\frac{\alpha-1}{2-\alpha} \cdot(1-\ln t)^{2-\alpha}\right]_{\rho}^{1}=-\infty
\end{gathered}
$$

The following lemma on the Newtonian potentials is important for obtaining solutions of a higher regularity to the Poisson equations as well as to the corresponding semi-linear equations. 
In this connection, recall the definition of the formal complex derivatives:

$$
\frac{\partial}{\partial z}:=\frac{1}{2}\left\{\frac{\partial}{\partial x}-i \cdot \frac{\partial}{\partial y}\right\}, \quad \frac{\partial}{\partial \bar{z}}:=\frac{1}{2}\left\{\frac{\partial}{\partial x}+i \cdot \frac{\partial}{\partial y}\right\}, \quad z=x+i y .
$$

The elementary algebraic calculations show their relation to the Laplacian

$$
\triangle:=\frac{\partial^{2}}{\partial x^{2}}+\frac{\partial^{2}}{\partial y^{2}}=4 \cdot \frac{\partial^{2}}{\partial z \partial \bar{z}}=4 \cdot \frac{\partial^{2}}{\partial \bar{z} \partial z}
$$

Further we apply the theory of the well-known integral operators

$$
T g(z):=\frac{1}{\pi} \int_{\mathbb{C}} g(w) \frac{d m(w)}{z-w}, \quad \bar{T} g(z):=\frac{1}{\pi} \int_{\mathbb{C}} g(w) \frac{d m(w)}{\bar{z}-\bar{w}}
$$

defined for integrable functions with a compact support $K$ and studied in detail. Recall the known results on them in Chapter 1 of [71], confining the case $K=\overline{\mathbb{D}}$, that are relevant to the proof of Theorem 2 .

First of all, if $g \in L^{1}(\mathbb{C})$, then by Theorem 1.13 the integrals $T g$ and $\bar{T} g$ exist a.e. in $\mathbb{C}$ and belong to $L_{\text {loc }}^{q}(\mathbb{C})$ for all $q \in[1,2)$ and by Theorem 1.14 they have generalized derivatives by Sobolev $(T g)_{\bar{z}}=g=(\bar{T} g)_{z}$. Furthermore, if $g \in L^{p}(\mathbb{C}), p>1$, then by Theorem 1.27 and (6.27) $T g$ and $\bar{T} g$ belong to $L_{\text {loc }}^{q}(\mathbb{C})$ for some $q>2$. and, moreover, by Theorems 1.36-1.37 $(T g)_{z}$ and $(\bar{T} g)_{\bar{z}}$ also belong to $L_{\text {loc }}^{p}(\mathbb{C})$. Finally, if $g \in L^{p}(\mathbb{C})$ for $p>2$, then by Theorem $1.19 \mathrm{Tg}$ and $\bar{T} g$ belong to $C_{\mathrm{loc}}^{\alpha}(\mathbb{C})$ with $\alpha=(p-2) / p$.

Here, given a domain $D$ in $\mathbb{C}$, a function $g: D \rightarrow \mathbb{R}$ is assumed to be extended onto $\mathbb{C}$ by zero outside of $D$.

Lemma 3. Let $D$ be a bounded domain in $\mathbb{C}$. Suppose that $g \in L^{1}(D)$. Then $N_{g} \in W_{\text {loc }}^{1, q}(\mathbb{C})$ for all $q \in[1,2)$ and there exist the generalized derivatives by Sobolev $\frac{\partial^{2} N_{g}}{\partial z \partial \bar{z}}$ and $\frac{\partial^{2} N_{g}}{\partial \bar{z} \partial z}$ and

$$
4 \cdot \frac{\partial^{2} N_{g}}{\partial z \partial \bar{z}}=\triangle N_{g}=4 \cdot \frac{\partial^{2} N_{g}}{\partial \bar{z} \partial z}=g \quad \text { a.e. in } \mathbb{C}
$$

Moreover, $N_{g} \in L_{\mathrm{loc}}^{s}(\mathbb{C})$ for all $s \in[1, \infty)$. More precisely,

$$
\left\|N_{g}\right\|_{s} \leq\|g\|_{1} \cdot\|\ln |\xi|\|_{s} \quad \forall s \in[1, \infty),
$$

where $\left\|N_{g}\right\|_{s}$ is in $\mathbb{D}_{r}$ for all $r \in(0, \infty)$ and $\|\ln |\xi|\|_{s}$ is in $\mathbb{D}_{R+r}$ if $D \subseteq \mathbb{D}_{R}$.

If $g \in L^{p}(D)$ for some $p \in(1,2]$, then $N_{g} \in W_{\text {loc }}^{2, p}(\mathbb{C})$ and

$$
N_{g} \in W_{\mathrm{loc}}^{1, \gamma}(\mathbb{C}) \quad \forall \gamma \in(1, q) \text {, where } q=2 p /(2-p)>2 .
$$


In addition, the collection $\left\{N_{g}\right\}$ is locally $\beta$-Hölder equicontinuous in $\mathbb{C}$ for all $\beta \in(0,1-2 / q)$ and the collection $\left\{N_{g}^{\prime}\right\}$ of its first partial derivatives is strictly compact in $L^{\gamma}(D)$ for all $\gamma \in(1, q)$ if the collection $\{g\}$ is bounded in $L^{p}(D)$.

Finally, if $g \in L^{p}(D)$ for some $p>2$, then $N_{g} \in C_{\text {loc }}^{1, \alpha}(\mathbb{C})$ with $\alpha=(p-2) / p$. Furthermore, the collection $\left\{N_{g}^{\prime}\right\}$ is locally Hölder equicontinuous in $\mathbb{C}$ with the given $\alpha$ if $\{g\}$ is bounded in $L^{p}(D)$.

Proof. Note that $N_{g}$ is the convolution $\psi * g$, where $\psi(\xi)=\ln |\xi|$, and hence (2.18) follows, e.g. by Corollary 4.5.2 in [46]. Moreover, $\frac{\partial \psi * g}{\partial z}=$ $\frac{\partial \psi}{\partial z} * g$ and $\frac{\partial \psi * g}{\partial \bar{z}}=\frac{\partial \psi}{\partial \bar{z}} * g$, see e.g. (4.2.5) in [46], and by elementary calculations

$$
\frac{\partial}{\partial z} \ln |z-w|=\frac{1}{2} \cdot \frac{1}{z-w}, \quad \frac{\partial}{\partial \bar{z}} \ln |z-w|=\frac{1}{2} \cdot \frac{1}{\bar{z}-\bar{w}} .
$$

Consequently,

$$
\frac{\partial N_{g}(z)}{\partial z}=\frac{1}{4} \cdot T g(z), \quad \frac{\partial N_{g}(z)}{\partial \bar{z}}=\frac{1}{4} \cdot \bar{T} g(z) .
$$

Thus, the rest conclusions for $g \in L^{1}(D)$ follow by Theorems 1.13-1.14 in [71].

Next, if $g \in L^{p}(D)$ with $p \in(1,2]$, then $N_{g} \in W_{\text {loc }}^{1, \gamma}(\mathbb{C})$ for all $\gamma \in$ $(1, q)$, where $q=2 p /(2-p)>2$, by Theorem 1.27, (1.27) in [71] and, moreover, $N_{g} \in W_{\text {loc }}^{2, p}(\mathbb{C})$ by Theorems $1.36-1.37$ in [71]. In addition, a collection $\left\{N_{g}\right\}$ is locally $\beta$-Hölder equicontinuous in $\mathbb{C}$ for all $\beta \in$ $(0,1-2 / q)$, see e.g. Lemma 2.7 in [15], and the collection $\left\{N_{g}^{\prime}\right\}$ of its first partial derivatives is strictly compact in $L^{\gamma}(D)$ for all $\gamma \in(1, q)$ if the collection $\{g\}$ is bounded by the norm in $L^{p}(D)$, see e.g. Theorem 1.4.3 in [69] and Theorem 1.27 in [71].

Finally, if $g \in L^{p}(D)$ for some $p>2$, then $N_{g} \in C_{\text {loc }}^{1, \alpha}(\mathbb{C})$ with $\alpha=$ $(p-2) / p$ by Theorem 1.19 in [71]. Furthermore, by the latter theorem the collection $\left\{N_{g}^{\prime}\right\}$ is also locally $\alpha$-Hölder equicontinuous in $\mathbb{C}$ with $\alpha=(p-2) / p$ if the collection $\{g\}$ is bounded by the norm in $L^{p}(D)$, $p>2$.

Remark 2. Note that generally speaking $N_{g} \notin W_{\text {loc }}^{2,1}$ for the case $g \in L^{1}(\mathbb{C})$, see e.g. example 7.5 in [25], p.141. Note also that the corresponding Newtonian potentials $N_{g}$ in $\mathbb{R}^{n}, n \geq 3$, also belong to $W_{\text {loc }}^{2, p}$ if $g \in L^{p}(\mathbb{C})$ for $p>1$ with compact support, see e.g. [26], Theorem 9.9.

As above, we assume here that $g: D \rightarrow \mathbb{R}$ is extended by zero outside of $D$. 
Corollary 2. Let $D$ be a subdomain of $\mathbb{D}, g: D \rightarrow \mathbb{R}$ be in $L^{1}(D)$ and in $L_{\mathrm{loc}}^{p}(D)$ for some $p>1$. Then $N_{g}$ satisfies $(2.17)$ a.e. in $D$. Moreover, $N_{g} \in W_{\text {loc }}^{1, q}(D)$ for $q>2$ and $N_{g}$ is locally Hölder continuous in D. Furthermore, $N_{g} \in C_{\mathrm{loc}}^{1, \alpha}(D)$ with $\alpha=(p-2) / p$ if $g \in L_{\mathrm{loc}}^{p}(D)$ for $p>2$.

In addition, the collection $\left\{N_{g}\right\}$ is locally $\beta$-Hölder equicontinuous in $D$ for all $\beta \in(0,1-2 / q)$ and the collection $\left\{N_{g}^{\prime}\right\}$ of its first partial derivatives is strictly compact in $L_{\mathrm{loc}}^{\gamma}(D)$ for all $\gamma \in(1, q)$ if a collection $\{g\}$ is bounded in $L^{1}(D)$ and in $L_{\mathrm{loc}}^{p}(D)$ for some $p \in(1,2]$, where $q$ is defined in (2.19).

Finally, the collection $\left\{N_{g}^{\prime}\right\}$ is locally $\alpha$-Hölder equicontinuous in $D$ with the given $\alpha$ if a collection $\{g\}$ is bounded in $L^{1}(D)$ and in $L_{\mathrm{loc}}^{p}(D)$ for $p>2$.

Proof. Given $z_{0} \in D$ and $0<R<\operatorname{dist}\left(z_{0}, \partial D\right), N_{g}=N_{g_{1}}+N_{g_{2}}$ with $g_{2}:=g-g_{1}$ and $g_{1}:=g \cdot \chi$ where $\chi$ is the characteristic function of the disk $\mathbb{D}_{R}\left(z_{0}\right)$. The first summand satisfies all desired properties by Lemma 3 and the second one is a harmonic function in $\mathbb{D}_{R}\left(z_{0}\right)$, see e.g. Theorem 3.1.2 in [64]. Thus, the first part follows. Under the proof of the rest part, it is applied the same decomposition. However, in the case we need the following 2 explicit estimates for the second summand in a smaller disk $\mathbb{D}_{r}\left(z_{0}\right), r \in(0, R)$,

$\left|N_{g_{2}}\left(z_{2}\right)-N_{g_{2}}\left(z_{1}\right)\right| \leq\left|\int_{z_{1}}^{z_{2}} \frac{\partial N_{g_{2}}}{\partial z} d z\right|+\left|\int_{z_{1}}^{z_{2}} \frac{\partial N_{g_{2}}}{\partial \bar{z}} d \bar{z}\right| \leq \frac{1}{2 \pi} \cdot \frac{\|g\|_{1}}{(R-r)} \cdot\left|z_{2}-z_{1}\right|$

and, since the function $T g_{2}$ is analytic in $\mathbb{D}_{r}\left(z_{0}\right)$ and the function $\bar{T} g_{2}=$ $\overline{T g_{2}}$ (for the real-valued function $\left.g_{2}\right)$ is anti-analytic in $\mathbb{D}_{r}\left(z_{0}\right)$, similarly

$$
\left|N_{g_{2}}^{\prime}\left(z_{2}\right)-N_{g_{2}}^{\prime}\left(z_{1}\right)\right| \leq \frac{1}{4}\left|\int_{z_{1}}^{z_{2}} \frac{\partial T g_{2}}{\partial z} d z\right| \leq \frac{1}{4 \pi} \cdot \frac{\|g\|_{1}}{(R-r)^{2}} \cdot\left|z_{2}-z_{1}\right| .
$$

Here we denote by $N_{g_{2}}^{\prime}$ any of the first partial derivatives of $N_{g_{2}}$, see (2.15):

$$
\frac{\partial}{\partial x}=\frac{\partial}{\partial z}+\frac{\partial}{\partial \bar{z}}, \quad \frac{\partial}{\partial y}=i \cdot\left(\frac{\partial}{\partial z}-\frac{\partial}{\partial \bar{z}}\right), \quad z=x+i y,
$$

take into account relation (2.20) and calculate the given integrals over the segment $\left[z_{1}, z_{2}\right] \subset D_{r}\left(z_{0}\right)$ of straight line going through $z_{1}, z_{2} \in$ $\mathbb{D}_{r}\left(z_{0}\right)$. 


\section{On solvability of quasilinear Poisson equations}

In this section we study the solvability problem for quasilinear Poisson equations of the form $\triangle U=h(z) f(U)$. The well-known Leray-Schauder approach allows us to reduce the problem to the study of the corresponding linear Poisson equation from the previous section.

For the sake of completeness, we recall some definitions and basic facts of the celebrated paper [56].

First of all, Leray and Schauder define a completely continuous mapping from a metric space $M_{1}$ into a metric space $M_{2}$ as a continuous mapping on $M_{1}$ which takes bounded subsets of $M_{1}$ into relatively compact ones of $M_{2}$, i.e. with compact closures in $M_{2}$. When a continuous mapping takes $M_{1}$ into a relatively compact subset of $M_{1}$, it is nowadays said to be compact on $M_{1}$.

Then Leray and Schauder extend as follows the Brouwer degree to compact perturbations of the identity $I$ in a Banach space $B$, i.e. a complete normed linear space. Namely, given an open bounded set $\Omega \subset B$, a compact mapping $F: B \rightarrow B$ and $z \notin \Phi(\partial \Omega), \Phi:=I-F$, the (LeraySchauder) topological degree $\operatorname{deg}[\Phi, \Omega, z]$ of $\Phi$ in $\Omega$ over $z$ is constructed from the Brouwer degree by approximating the mapping $F$ over $\Omega$ by mappings $F_{\varepsilon}$ with range in a finite-dimensional subspace $B_{\varepsilon}$ (containing $z$ ) of $B$. It is showing that the Brouwer degrees $\operatorname{deg}\left[\Phi_{\varepsilon}, \Omega_{\varepsilon}, z\right]$ of $\Phi_{\varepsilon}:=I_{\varepsilon}-F_{\varepsilon}, I_{\varepsilon}:=\left.I\right|_{B_{\varepsilon}}$, in $\Omega_{\varepsilon}:=\Omega \cap B_{\varepsilon}$ over $z$ stabilize for sufficiently small positive $\varepsilon$ to a common value defining $\operatorname{deg}[\Phi, \Omega, z]$ of $\Phi$ in $\Omega$ over $z$.

This topological degree "algebraically counts" the number of fixed points of $F(\cdot)-z$ in $\Omega$ and conserves the basic properties of the Brouwer degree as additivity and homotopy invariance. Now, let $a$ be an isolated fixed point of $F$. Then the local (Leray-Schauder) index of $a$ is defined by ind $[\Phi, a]:=\operatorname{deg}[\Phi, B(a, r), 0]$ for small enough $\mathrm{r}>0$. If $a=0$, then we say on the index of $F$. In particular, if $F \equiv 0$, correspondingly, $\Phi \equiv I$, then the index of $F$ is equal to 1.

The fundamental Theorem 1 in [56] can be formulated in the following way: Let $B$ be a Banach space, and let $F(\cdot, \tau): B \rightarrow B$ be a family of operators with $\tau \in[0,1]$. Suppose that the following hypotheses hold:

(H1) $F(\cdot, \tau)$ is completely continuous on $B$ for each $\tau \in[0,1]$ and uniformly continuous with respect to the parameter $\tau \in[0,1]$ on each bounded set in $B$;

(H2) the operator $F:=F(\cdot, 0)$ has finite collection of fixed points whose total index is not equal to zero; 
(H3) the collection of all fixed points of the operators $F(\cdot, \tau), \tau \in$ $[0,1]$, is bounded in $B$.

Then the collection of all fixed points of the family of operators $F(\cdot, \tau)$ contains a continuum along which $\tau$ takes all values in $[0,1]$.

In the proof of the next theorem the initial operator $F(\cdot):=F(\cdot, 0) \equiv$ 0 . Hence $F$ has the only one fixed point (at the origin) and its index is equal to 1 and, thus, hypothesis (H2) will be automatically satisfied.

Theorem 1. Let $h: \mathbb{C} \rightarrow \mathbb{R}$ be a function in the class $L^{p}(\mathbb{C})$ for $p>1$ with compact support. Suppose that a function $f: \mathbb{R} \rightarrow \mathbb{R}$ is continuous and

$$
\lim _{t \rightarrow \infty} \frac{f(t)}{t}=0 \text {. }
$$

Then there is a continuous function $U: \mathbb{C} \rightarrow \mathbb{R}$ in the class $W_{\text {loc }}^{2, p}(\mathbb{C})$ such that

$$
\triangle U(z)=h(z) \cdot f(U(z)) \quad \text { a.e. }
$$

and $U=N_{g}$ where $g: \mathbb{C} \rightarrow \mathbb{R}$ is a function in $L^{p}$ whose support is in the support of $h$ and the upper bound of $\|g\|_{p}$ depends only on $\|h\|_{p}$ and on the function $f$. Moreover, $U \in W_{\mathrm{loc}}^{1, q}(\mathbb{C})$ for some $q>2$ and $U$ is locally Hölder continuous. Furthermore, $U \in C_{\mathrm{loc}}^{1, \alpha}(\mathbb{C})$ with $\alpha=(p-2) / p$ if $p>2$.

In particular, $U \in C_{\text {loc }}^{1, \alpha}(\mathbb{C})$ for all $\alpha=(0,1)$ if $h$ is bounded in Theorem 1.

Proof. If $\|h\|_{p}=0$ or $\|f\|_{C}=0$, then any constant function $U$ in $\mathbb{C}$ gives the desired solution of (3.2). Thus, we may assume that $\|h\|_{p} \neq 0$ and $\|f\|_{C} \neq 0$. Set $f_{*}(s)=\max _{|t| \leq s}|f(t)|, s \in \mathbb{R}^{+}:=[0, \infty)$. Then the function $f_{*}: \mathbb{R}^{+} \rightarrow \mathbb{R}^{+}$is continuous and nondecreasing and, moreover, $f_{*}(s) / s \rightarrow 0$ as $s \rightarrow \infty$ by $(3.1)$.

By Lemma 2 we obtain the family of operators $F(g ; \tau): L_{h}^{p}(\mathbb{C}) \rightarrow$ $L_{h}^{p}(\mathbb{C})$, where $L_{h}^{p}(\mathbb{C})$ consists of functions $g \in L^{p}(\mathbb{C})$ with supports in the support of $h$,

$$
F(g ; \tau):=\tau h \cdot f\left(N_{g}\right) \quad \forall \tau \in[0,1]
$$

which satisfies all groups of hypothesis H1-H3 of Theorem 1 in [56]. Indeed:

H1). First of all, $F(g ; \tau) \in L_{h}^{p}(\mathbb{C})$ for all $\tau \in[0,1]$ and $g \in L_{h}^{p}(\mathbb{C})$ because by Lemma 2 the function $f\left(N_{g}\right)$ is continuous and

$$
\|F(g ; \tau)\|_{p} \leq\|h\|_{p} f_{*}\left(M\|g\|_{p}\right)<\infty \quad \forall \tau \in[0,1],
$$


where $M$ is the constant from the estimate (2.14). Thus, by Lemma 2 in combination with the Arzela-Ascoli theorem, see e.g. Theorem IV.6.7 in [17], the operators $F(g ; \tau)$ are completely continuous for each $\tau \in[0,1]$ and even uniformly continuous with respect to the parameter $\tau \in[0,1]$.

H2). The index of the operator $F(g ; 0)$ is obviously equal to 1 .

H3). By Lemma 2 we have also for solutions of the equations $g=$ $F(g ; \tau)$ :

$$
\|g\|_{p} \leq\|h\|_{p} f_{*}\left(M\|g\|_{p}\right)
$$

i.e.,

$$
\frac{f_{*}\left(M\|g\|_{p}\right)}{M\|g\|_{p}} \geq \frac{1}{M\|h\|_{p}}
$$

and hence $\|g\|_{p}$ should be bounded in view of condition (3.1).

Thus, by Theorem 1 in [56] there is a function $g \in L_{h}^{p}(D)$ with $F(g ; 1)=g$, and by Lemma 3 the function $U:=N_{g}$ gives the desired solution of (3.2).

Corollary 3. Let $D$ be a subdomain of $\mathbb{D}, h: D \rightarrow \mathbb{R}$ be in $L^{1}(D)$ and in $L_{\mathrm{loc}}^{p}(D)$ for some $p>1$. Suppose that a function $f: \mathbb{R} \rightarrow \mathbb{R}$ satisfies the hypothesis of Theorem 1. Then there is a weak solution $u: D \rightarrow \mathbb{R}$ of the quasilinear Poisson equation (3.2) which is locally Hölder continuous in $D$.

Proof. Let $D_{k}$ be an expanding sequence of domains in $\mathbb{C}$ with $\overline{D_{k}} \subset D$, $k=1,2, \ldots$, exhausting $D$, i.e. $\bigcup_{k=1}^{\infty} D_{k}=D$. Let us extend $h$ by zero outside of $D$. Set $h_{k}=h \chi_{k}$, where $\chi_{k}$ is a characteristic function of $D_{k}$ in $\mathbb{C}$, and $U_{k}=N_{g_{k}}$, where $g_{k}$ corresponds to $h_{k}$ by Theorem 1 . Note that the sequence $\left\|g_{k}\right\|_{p}, k=1,2, \ldots$, is bounded on each $D_{m}, m=1,2, \ldots$, by Theorem 1 . Hence by Lemma 2 the sequence $\left|N_{g_{k}}\right|_{C}$ is also bounded on each $D_{m}, m=1,2, \ldots$. Now, by Corollary 2 the family of functions $\left\{N_{g_{k}}\right\}$ is Hölder equicontinuous on each $D_{m}, m=1,2, \ldots$. Thus, by the Arzela-Ascoli theorem, see e.g. Theorem IV.6.7 in [17], the family of functions $\left\{N_{g_{k}}\right\}$ is compact on each $D_{m}, m=1,2, \ldots$.

Without loss of generality, we may assume that $p \in(1,2]$. Then by Corollary 2 the Newtonian potential $\left\{N_{g_{k}}\right\}, m=1,2, \ldots$, is in the class $W_{\text {loc }}^{1, q}$ for some $q>2$ and the family $\left\{N_{g_{k}}^{\prime}\right\}$ is also compact on each $D_{m}$, $m=1,2, \ldots$ by the norm of $L^{q}$. Consequently, the sequence $\left\{N_{g_{k}}\right\}$ is compact on each $D_{m}, m=1,2, \ldots$ by any norm $\|\cdot\|$ of $W^{1, q}$, too, see e.g. Theorem 2.5.1 in [59].

Next, let us apply the so-called diagonal process. Namely, let $u_{k}^{(1)}$, $k=1,2, \ldots$, be a subsequence of $\left\{N_{g_{k}}\right\}$ that converges uniformly and by 
the norm $\|\cdot\|$ on the domain $D_{1}$ to a function $u: D_{1} \rightarrow \mathbb{R}$. Of course, we may assume that $\left\|u_{k}^{(1)}-u\right\|_{C}<1 / k$ as well as $\left\|u_{k}^{(1)}-u\right\|<1 / k$ for all $k=1,2, \ldots$. Similarly, it is defined a subsequence $u_{k}^{(2)}$ of $u_{k}^{(1)}$ with respect to the domain $D_{2}$. Let us continue the process by induction and, finally, consider the diagonal subsequence $u_{m}:=u_{m}^{(m)}, m=1,2, \ldots$ of the sequence $N_{g_{k}}$.

It is clear by the construction that $\left.u_{m}\right|_{D}$ converges to a function $u: D \rightarrow \mathbb{R}$ locally uniformly and in $W_{\text {loc }}^{1, q}(D), q>2$. Thus, $u \in C(D) \cap W_{\text {loc }}^{1, q}(D)$ and, consequently, $u$ is locally Hölder continuous in $D$. Moreover, $u$ is a weak solution of the equation (3.2) in the domain $D$. Indeed, by Corollary 1 and the definition of generalized derivatives, we have that $u_{m}$ satisfy the relations

$$
\begin{gathered}
\int_{D}\left\langle\nabla u_{m}(z), \nabla \psi(z)\right\rangle d m(z)+\int_{D} h_{m}(z) f\left(u_{m}(z)\right) \psi(z) d m(z) \\
=0 \quad \forall \psi \in C_{0}^{\infty}(D)
\end{gathered}
$$

and, passing to the limit as $m \rightarrow \infty$, we obtain the desired conclusion.

\section{On solvability of semi-linear equations}

In this section we study the solvability problem for the semi-linear equations of the form $\operatorname{div}[A(z) \nabla u]=f(u)$. Following the paper [36], under a weak solution to this equation we understand a function $u \in$ $C(D) \cap W_{\text {loc }}^{1,2}(D)$ such that

$$
\int_{D}\langle A(z) \nabla u(z), \nabla \varphi(z)\rangle d m(z)+\int_{D} f(u(z)) \varphi(z) d m(z)=0
$$

for all $\varphi \in C(D) \cap W_{0}^{1,2}(D)$.

Theorem 2. Let $D$ be a domain in $\mathbb{C}$ with a finite area that is not dense in $\mathbb{C}$. Suppose that $A \in M_{K}^{2 \times 2}(D)$ and a continuous function $f: \mathbb{R} \rightarrow \mathbb{R}$ satisfies condition (3.1). Then there is a weak solution $u: D \rightarrow \mathbb{R}$ of equation

$$
\operatorname{div}[A(z) \nabla u(z)]=f(u),
$$

which is locally Hölder continuous in D.

Proof. Let us extend by definition $A \equiv I$ (the unit matrix) outside of $D$. By Theorem 4.1 in [36], if $u$ is a weak solution of (4.2), then $u=U \circ \omega$, 
where $\omega:=\left.\Omega\right|_{D}$ and $\Omega$ is a quasiconformal mapping of $\overline{\mathbb{C}}$ onto itself, $\Omega(\infty)=\infty$, agreed with the extended $A$, and $U$ is a weak solution of (3.2) with $h=J$, where $J$ is the Jacobian of the mapping $\omega^{-1}: D_{*} \rightarrow D$, $D_{*}:=\omega(D)$.

Note that $\overline{\mathbb{C}} \backslash D$ contains a nondegenerate (connected) component $C$ because of $D$ is not dense in $\mathbb{C}$, see e.g. Corollary IV.2 and the point II.4.D in [47], see also Lemma 5.1 in [48] or Lemma 6.3 in [57]. Hence $\overline{\mathbb{C}} \backslash$ $D_{*}$ contains a component $C_{*}:=\Omega(C)$ whose boundary is a nondegenerate continuum, see again Lemma 5.1 in [48] or Lemma 6.3 in [57], and by the Riemann theorem there is a conformal mapping $H$ of $\overline{\mathbb{C}} \backslash C_{*}$ onto $\mathbb{D}$.

Setting $H_{*}=\left.H\right|_{D_{*}}$, we see that $H_{*}$ maps $D_{*}$ into $\mathbb{D}$. Moreover, the quasiconformal mapping $\omega_{*}:=H_{*} \circ \omega: D \rightarrow \mathbb{D}_{*}:=H_{*}\left(D_{*}\right)$ is also agreed with $A$ in $D$. Thus, again by Theorem 4.1 in [36], $u=U_{*} \circ \omega_{*}$ where $U_{*}$ is a weak solution of (3.2) with $h=J_{*}$ in $\mathbb{D}_{*} \subseteq \mathbb{D}$, here $J_{*}$ is the Jacobian of the mapping $\omega_{*}^{-1}: \mathbb{D}_{*} \rightarrow D$.

Also by Remark 4.1 in [36], inversely, if $U_{*}$ is a weak solution of (3.2) with $h=J_{*}$ in $\mathbb{D}_{*}$, then $u:=U_{*} \circ \omega_{*}$ is a weak solution of (4.2) in $D$. The latter implication allows us to reduce the proof of Theorem 2 to Corollary 3 with the special $h=J_{*}$.

Indeed, $J_{*} \in L^{1}\left(\mathbb{D}_{*}\right)$ because its integral is equal to the area of the domain $D$, see e.g Theorem 3.2 in [12] and Theorem II.B.3 in [1]. Moreover, $J_{*} \in L_{\mathrm{loc}}^{p}\left(\mathbb{D}_{*}\right)$ for some $p>1$ because by the Bojarski result, see [11] and [12], the first partial derivatives of the quasiconformal mapping $\omega^{*}:=\omega_{*}^{-1}: \mathbb{D}_{*} \rightarrow D$ are locally integrable with a power $q>2$ and $J_{*}=\left|\omega_{w}^{*}\right|^{2}-\left|\omega_{\bar{w}}^{*}\right|^{2}$, see e.g. I.A(9) in [1].

Remark 3. Note that it is easy to construct a set $C$ in $\mathbb{C}$ of the Cantor type which is dense in the plane $\mathbb{C}$ whose completion has a finite area, furthermore, an arbitrarily small area.

Indeed, let us cover the plane by a collection $\mathcal{S}$ plates consisting of closed squares with unit sides oriented along coordinate axes $x$ and $y$, $z=x+i y \in \mathbb{C}$, that can intersect each other only by their common sides. Let $S_{n}, n=1,2, \ldots$, be some enumeration of the squares in $\mathcal{S}$ and let $\varepsilon \in(0,1)$ be arbitrary.

First, let us remove narrow symmetric strips of the same width in $S_{1}$ along its sides whose total area is less that $\varepsilon / 4$. We have in the rest a central square. Then let us cut out narrow centralized horizontal and vertical corridors of the same width in the last square whose total area is less than $\varepsilon / 8$. These corridors form a cross that splits the last square into 4 squares. In turn, we remove from these squares similar crosses of the total area $\varepsilon / 16$ that split them on the whole into $4^{2}$ squares. Repeating the procedure by induction, we remove from $S_{1}$ corridors with the total 
area $\varepsilon / 2$ and the intersection of all mentioned squares gives a totally disconnected compactum $C_{1} \neq \emptyset$ of the Cantor type, see e.g. $4.41\left(2^{\prime}\right)$ in [50].

Similarly, we are able to construct such a set $C_{n} \subset S_{n}$ with its completion in $S_{n}$ whose area is less than $\varepsilon / 2^{1+n}$ for each $n=1,2, \ldots$ Then the set $C:=\bigcup_{n=1}^{\infty} C_{n}$ has the completion in $\mathbb{C}$ whose area is less than $\varepsilon$. Note that by our construction the set $C$ is totally disconnected and, thus, its topological dimension is equal to 0, see e.g. Proposition II.4.D in [47]. It is clear that $D:=\mathbb{C} \backslash C$ is a domain, see e.g. Theorem IV.4 in [47].

Finally, note that our example of the set $C$ of the Cantor type in the plane with its topological dimension 0 is essentially different from the well-known Sierpinski cover whose topological dimension is equal to 1.

\section{The Dirichlet problem with continuous data for quasilinear Poisson equations}

Let $D$ be a bounded domain in $\mathbb{C}$ without degenerate boundary components, i.e., any connected component of the boundary of $D$ is not degenerated to a single point. Given a continuous boundary function $\varphi: \partial D \rightarrow \mathbb{R}$, let us denote by $\mathcal{D}_{\varphi}$ the harmonic function in $D$ that has the continuous extension to $\bar{D}$ with $\varphi$ as its boundary date. Such a function exists and it is unique, see e.g. Corollary 4.1.8 and Theorem 4.2.2 in [64]. Thus, the Dirichlet operator $\mathcal{D}_{\varphi}$ is well defined in the given domains. We need not its explicit description for our goals.

By Lemma 3 we come to the following result on the existence, regularity and representation of solutions for the Dirichlet problem to the Poisson equation in arbitrary bounded domains $D$ in $\mathbb{C}$ without degenerate boundary components where we assume that the charge density $g$ is extended by zero outside of $D$.

Theorem 3. Let $D$ be a bounded domain in $\mathbb{C}$ without degenerate boundary components, $\varphi: \partial D \rightarrow \mathbb{R}$ be a continuous function and $g:$ $D \rightarrow \mathbb{R}$ belong to the class $L^{p}(D)$ for $p>1$. Then the function

$$
U:=N_{g}-\mathcal{D}_{N_{g}^{*}}+\mathcal{D}_{\varphi}, \quad N_{g}^{*}:=\left.N_{g}\right|_{\partial D},
$$

is continuous in $\bar{D}$ with $\left.U\right|_{\partial D}=\varphi$, belongs to the class $W_{\mathrm{loc}}^{2, p}(D)$ and satisfies the Poisson equation $\triangle U=g$ a.e. in D. Moreover, $U \in W_{\mathrm{loc}}^{1, q}(D)$ for some $q>2$ and $U$ is locally Hölder continuous in D. Furthermore, $U \in C_{\mathrm{loc}}^{1, \alpha}(D)$ with $\alpha=(p-2) / p$ if $g \in L^{p}(D)$ for $p>2$. 
Remark 4. Note also by the way that a generalized solution of the Dirichlet problem to the Poisson equation in the class $C(\bar{D}) \cap W_{\text {loc }}^{1,2}(D)$ is unique at all, see e.g. Theorem 8.30 in [26], and (5.1) gives the effective representation of this unique solution.

The case of quasilinear Poisson equations is reduced to the case of the linear Poisson equations again by the Leray-Schauder approach as in the last section.

Theorem 4. Let $D$ be a bounded domain in $\mathbb{C}$ without degenerate boundary components, $\varphi: \partial D \rightarrow \mathbb{R}$ be a continuous function and $h$ : $D \rightarrow \mathbb{R}$ be a function in the class $L^{p}(D)$ for $p>1$. Suppose that a function $f: \mathbb{R} \rightarrow \mathbb{R}$ is continuous and

$$
\lim _{t \rightarrow \infty} \frac{f(t)}{t}=0
$$

Then there is a continuous function $U: \bar{D} \rightarrow \mathbb{R}$ with $\left.U\right|_{\partial D}=\varphi$ and $\left.U\right|_{D} \in W_{\text {loc }}^{2, p}$ such that

$$
\triangle U(z)=h(z) \cdot f(U(z)) \quad \text { for a.e. } z \in D .
$$

Moreover, $U \in W_{\text {loc }}^{1, q}(D)$ for some $q>2$ and $U$ is locally Hölder continuous. Furthermore, $U \in C_{\mathrm{loc}}^{1, \alpha}(D)$ with $\alpha=(p-2) / p$ if $p>2$.

In particular, the latter statement in Theorem 4 implies that $U \in$ $C_{\text {loc }}^{1, \alpha}(D)$ for all $\alpha=(0,1)$ if $h$ is bounded.

Proof. If $\|h\|_{p}=0$ or $\|f\|_{C}=0$, then the Dirichlet operator $\mathcal{D}_{\varphi}$ gives the desired solution of the Dirichlet problem for equation (5.3), see e.g. I.D.2 in [49]. Hence we may assume further that $\|h\|_{p} \neq 0$ and $\|f\|_{C} \neq 0$. Set $f_{*}(s)=\max _{|t| \leq s}|f(t)|, s \in \mathbb{R}^{+}$. Then the function $f_{*}: \mathbb{R}^{+} \rightarrow \mathbb{R}^{+}$is continuous and nondecreasing and, moreover, $f_{*}(s) / s \rightarrow 0$ as $s \rightarrow \infty$ by (5.2).

By Lemma 2 and the maximum principle for harmonic functions, we obtain the family of operators $F(g ; \tau): L^{p}(D) \rightarrow L^{p}(D), \tau \in[0,1]$ :

$$
F(g ; \tau):=\tau h \cdot f\left(N_{g}-\mathcal{D}_{N_{g}^{*}}+\mathcal{D}_{\varphi}\right), N_{g}^{*}:=\left.N_{g}\right|_{\partial D}, \quad \forall \tau \in[0,1]
$$

which satisfies all groups of hypothesis H1-H3 of Theorem 1 in [56]. Indeed:

H1). First of all, $F(g ; \tau) \in L^{p}(D)$ for all $\tau \in[0,1]$ and $g \in L^{p}(D)$ because by Lemma $2 f\left(N_{g}-\mathcal{D}_{N_{g}^{*}}+\mathcal{D}_{\varphi}\right)$ is a continuous function and, moreover,

$$
\|F(g ; \tau)\|_{p} \leq\|h\|_{p} f_{*}\left(2 M\|g\|_{p}+\|\varphi\|_{C}\right)<\infty \quad \forall \tau \in[0,1] .
$$


Thus, by Lemma 2 in combination with the Arzela-Ascoli theorem, see e.g. Theorem IV.6.7 in [17], the operators $F(g ; \tau)$ are completely continuous for each $\tau \in[0,1]$ and even uniformly continuous with respect to the parameter $\tau \in[0,1]$.

H2). The index of the operator $F(g ; 0)$ is obviously equal to 1 .

H3). By Lemma 2 and the maximum principle for harmonic functions, we have the estimate for solutions $g \in L^{p}$ of the equations $g=F(g ; \tau)$

$$
\|g\|_{p} \leq\|h\|_{p} f_{*}\left(2 M\|g\|_{p}+\|\varphi\|_{C}\right) \leq\|h\|_{p} f_{*}\left(3 M\|g\|_{p}\right)
$$

whenever $\|g\|_{p} \geq\|\varphi\|_{C} / M$, i.e. then it should be

$$
\frac{f_{*}\left(3 M\|g\|_{p}\right)}{3 M\|g\|_{p}} \geq \frac{1}{3 M\|h\|_{p}}
$$

and hence $\|g\|_{p}$ should be bounded in view of condition (5.2).

Thus, by Theorem 1 in [56] there is a function $g \in L^{p}(D)$ such that $g=F(g ; 1)$ and, consequently, by Lemma 3 the function $U:=N_{g}-$ $\mathcal{D}_{N_{g}^{*}}+\mathcal{D}_{\varphi}$ gives the desired solution of the Dirichlet problem for the quasilinear Poisson equation (5.3).

Remark 5. As it is clear from the proof, condition (5.2) can be replaced by the following weaker condition with $M$ from the estimate in Lemma 2

$$
\limsup _{s \rightarrow \infty} \frac{f_{*}(s)}{s}<\frac{1}{3 M\|h\|_{p}} .
$$

Theorem 4 can be applied to some physical problems. The first circle of such applications is relevant to reaction-diffusion problems. Problems of this type are discussed in [16], p. 4, and, in detail, in [3]. A nonlinear system is obtained for the density $u$ and the temperature $T$ of the reactant. Upon eliminating $T$ the system can be reduced to the equation

$$
\triangle u=\lambda \cdot f(u)
$$

with $h(z) \equiv \lambda>0$ and, for isothermal reactions, $f(u)=u^{q}$ where $q>0$ is called the order of the reaction. It turns out that the density of the reactant $u$ may be zero in a subdomain called a dead core. A particularization of results in Chapter 1 of [16] shows that a dead core may exist just if and only if $0<q<1$ and $\lambda$ is large enough, see also the corresponding examples in [36]. In this connection, the following statements may be of independent interest. 
Corollary 4. Let $D$ be a bounded domain in $\mathbb{C}$ without degenerate boundary components, $\varphi: \partial D \rightarrow \mathbb{R}$ be a continuous function and let $h: D \rightarrow \mathbb{R}$ be a function in the class $L^{p}(D), p>1$. Then there exists a continuous function $u: \bar{D} \rightarrow \mathbb{R}$ with $\left.u\right|_{\partial D}=\varphi$ such that $u \in W_{\mathrm{loc}}^{2, p}(D)$ and

$$
\triangle u(z)=h(z) \cdot u^{q}(z), \quad 0<q<1
$$

a.e. in D. Moreover, $u \in W_{\mathrm{loc}}^{1, \beta}(D)$ for some $\beta>2$ and $u$ is locally Hölder continuous in D. Furthermore, $u \in C_{\text {loc }}^{1, \alpha}(D)$ with $\alpha=(p-2) / p$ if $p>2$.

Corollary 5. Let $D$ be a bounded domain in $\mathbb{C}$ without degenerate boundary components and $\varphi: \partial D \rightarrow \mathbb{R}$ be a continuous function. Then there is a continuous function $u: \bar{D} \rightarrow \mathbb{R}$ with $\left.u\right|_{\partial D}=\varphi$ such that $u \in C_{\mathrm{loc}}^{1, \alpha}(D)$ for all $\alpha \in(0,1), u \in W_{\mathrm{loc}}^{2, p}(D)$ for all $p \in[1, \infty)$ and

$$
\triangle u(z)=u^{q}(z), \quad 0<q<1, \quad \text { a.e. in } D .
$$

Note also that certain mathematical models of a thermal evolution of a heated plasma lead to nonlinear equations of the type (5.7). Indeed, it is known that some of them have the form $\Delta \psi(u)=f(u)$ with $\psi^{\prime}(0)=\infty$ and $\psi^{\prime}(u)>0$ if $u \neq 0$ as, for instance, $\psi(u)=|u|^{q-1} u$ under $0<q<1$, see e.g. [16]. With the replacement of the function $U=\psi(u)=|u|^{q} \cdot \operatorname{sign} u$, we have that $u=|U|^{Q} \cdot \operatorname{sign} U, Q=1 / q$, and, with the choice $f(u)=$ $|u|^{q^{2}} \cdot \operatorname{sign} u$, we come to the equation $\triangle U=|U|^{q} \cdot \operatorname{sign} U=\psi(U)$.

Corollary 6. Let $D$ be a bounded domain in $\mathbb{C}$ without degenerate boundary components and $\varphi: \partial D \rightarrow \mathbb{R}$ be a continuous function. Then there is a continuous function $U: \bar{D} \rightarrow \mathbb{R}$ with $\left.U\right|_{\partial D}=\varphi$ such that $U \in C_{\mathrm{loc}}^{1, \alpha}(D)$ for all $\alpha \in(0,1), u \in W_{\mathrm{loc}}^{2, p}(D)$ for all $p \in[1, \infty)$ and

$$
\triangle U(z)=|U(z)|^{q-1} U(z), \quad 0<q<1, \quad \text { a.e. in } D \text {. }
$$

Finally, we recall that in the combustion theory, see e.g. [5], [62] and the references therein, the following model equation

$$
\frac{\partial u(z, t)}{\partial t}=\frac{1}{\delta} \cdot \Delta u+e^{u}, \quad t \geq 0, z \in D
$$

takes a special place. Here $u \geq 0$ is the temperature of the medium and $\delta$ is a certain positive parameter. We restrict ourselves here by the stationary case, although our approach makes it possible to study the 
parabolic equation (5.11), see [36]. Namely, the equation (5.3) is appeared here with $h \equiv \delta>0$ and the function $f(u)=e^{-u}$ that is bounded.

Corollary 7. Let $D$ be a bounded domain in $\mathbb{C}$ without degenerate boundary components and $\varphi: \partial D \rightarrow \mathbb{R}$ be a continuous function. Then there is a continuous function $U: \bar{D} \rightarrow \mathbb{R}$ with $\left.U\right|_{\partial D}=\varphi$ such that $U \in C_{\mathrm{loc}}^{1, \alpha}(D)$ for all $\alpha \in(0,1), u \in W_{\mathrm{loc}}^{2, p}(D)$ for all $p \in[1, \infty)$ and

$$
\triangle U(z)=\delta \cdot e^{U(z)}, \text { a.e. in } D \text {. }
$$

Due to the factorization theorem in [36], we extend these results to semi-linear equations describing the corresponding physical phenomena in anisotropic and inhomogeneous media in arbitrary bounded domain without degenerate boundary components, see the next section.

\section{The Dirichlet problem with continuous data for semi-linear equations}

By the factorization theorem from [36], mentioned in Introduction, the study of semi-linear equations (4.2) in bounded domains without degenerate boundary componentss $D$ is reduced, by means of a suitable quasiconformal change of variables, to the study of the corresponding quasilinear Poisson equations (5.3).

Theorem 5. Let $D$ be a bounded domain in $\mathbb{C}$ without degenerate boundary components, $A \in M_{K}^{2 \times 2}(D), \varphi: \partial D \rightarrow \mathbb{R}$ be an arbitrary continuous function, $f: \mathbb{R} \rightarrow \mathbb{R}$ be a continuous function such that

$$
\lim _{t \rightarrow \infty} \frac{f(t)}{t}=0 \text {. }
$$

Then there is a weak solution $u: D \rightarrow \mathbb{R}$ of the class $C(D) \cap W_{\text {loc }}^{1,2}(D)$ to the equation

$$
\operatorname{div}[A(z) \nabla u]=f(u)
$$

which is locally Hölder continuous in $D$ and continuous in $\bar{D}$ with $\left.u\right|_{\partial D}=$ $\varphi$.

Proof. Let us extend by definition $A \equiv I$ outside of $D$. By Theorem 4.1 in [36], if $u$ is a weak solution of the equation, then $u=U \circ \omega$, where $\omega:=\left.\Omega\right|_{D}$ and $\Omega$ is a quasiconformal mapping of $\mathbb{C}$ onto itself agreed with the extended $A$, and $U$ is a weak solution of the equation (5.3) with $h=J$, where $J$ is the restriction of the Jacobian of the mapping $\Omega^{-1}: \mathbb{C} \rightarrow \mathbb{C}$ to the domain $D_{*}:=\Omega(D)$. 
Inversely, by Remark 4.1 in [36], we see that if $U$ is a weak solution of (5.3) with $h=J$, then $u=U \circ \omega$ is a weak solution of our equation. The latter allows us to reduce Theorem 5 to Theorem 4 . Indeed, $\overline{D_{*}}=\Omega(\bar{D})$ is compact and by the celebrated Bojarski result, see [11] and [12], the generalized derivatives of the quasiconformal mapping $\Omega^{*}:=\Omega^{-1}: \mathbb{C} \rightarrow$ $\mathbb{C}$ are locally integrable with some power $q>2$. Note also that the Jacobian $J$ of its restriction $\omega^{*}:=\left.\Omega^{*}\right|_{D_{*}}$ is equal to $\left|\omega_{w}^{*}\right|^{2}-\left|\omega_{\bar{w}}^{*}\right|^{2}$, see e.g. I.A(9) in [1]. Consequently, $J \in L^{p}\left(D_{*}\right)$ for some $p>1$.

Specifying the reaction term $f(u)$ of the semi-linear equation, we arrive at the following statements concerning some concrete problems of the mathematical physics in inhomogeneous and anisotropic media.

Corollary 8. Let $D$ be a bounded domain in $\mathbb{C}$ without degenerate boundary components, $A \in M_{K}^{2 \times 2}(D)$ and $\varphi: \partial D \rightarrow \mathbb{R}$ be a continuous function. Then there is a continuous function $u: \bar{D} \rightarrow \mathbb{R}$ with $\left.u\right|_{\partial D}=\varphi$ which is locally Hölder continuous in $D$ and it is a weak solution in $D$ for the equation

$$
\operatorname{div}[A(z) \nabla u(z)]=u^{q}(z), \quad 0<q<1 .
$$

Corollary 9. Let $D$ be a bounded domain in $\mathbb{C}$ without degenerate boundary components, $A \in M_{K}^{2 \times 2}(D)$ and $\varphi: \partial D \rightarrow \mathbb{R}$ be a continuous function. Then there is a continuous function $u: \bar{D} \rightarrow \mathbb{R}$ with $\left.u\right|_{\partial D}=\varphi$ which is locally Hölder continuous in $D$ and it is a weak solution in $D$ for the equation

$$
\operatorname{div}[A(z) \nabla u(z)]=|u(z)|^{q-1} u(z), \quad 0<q<1 .
$$

Corollary 10. Let $D$ be a bounded domain in $\mathbb{C}$ without degenerate boundary components, $A \in M_{K}^{2 \times 2}(D)$ and $\varphi: \partial D \rightarrow \mathbb{R}$ be a continuous function. Then there is a continuous function $u: \bar{D} \rightarrow \mathbb{R}$ with $\left.u\right|_{\partial D}=\varphi$ which is locally Hölder continuous in $D$ and it is a weak solution in $D$ for the equation

$$
\operatorname{div}[A(z) \nabla u(z)]=e^{\alpha u(z)}, \quad \alpha \in \mathbb{R} .
$$

Note that the statements given above remain hold if the reaction terms in equations (6.2)-(6.4) are multiplied by functions $C \in L^{\infty}(D)$.

The rest of the paper we are going to devote to the study of the Dirichlet problem for the Poisson equation with measurable boundary data and start with the notion of the logarithmic capacity. 


\section{The definition and preliminary remarks on the logarithmic capacity}

Given a bounded Borel set $E$ in the plane $\mathbb{C}$, a mass distribution on $E$ is a nonnegative completely additive function $\nu$ of a set defined on its Borel subsets with $\nu(E)=1$. The function

$$
U^{\nu}(z):=\int_{E} \log \left|\frac{1}{z-\zeta}\right| d \nu(\zeta)
$$

is called a logarithmic potential of the mass distribution $\nu$ at a point $z \in \mathbb{C}$. A logarithmic capacity $C(E)$ of the Borel set $E$ is the quantity

$$
C(E)=e^{-V}, \quad V=\inf _{\nu} V_{\nu}(E), \quad V_{\nu}(E)=\sup _{z} U^{\nu}(z) .
$$

It is also well-known the following geometric characterization of the logarithmic capacity, see e.g. the point 110 in [61]:

$$
C(E)=\tau(E):=\lim _{n \rightarrow \infty} V_{n}^{\frac{2}{n(n-1)}}
$$

where $V_{n}$ denotes the supremum of the product

$$
V\left(z_{1}, \ldots, z_{n}\right)=\prod_{k<l}^{l=1, \ldots, n}\left|z_{k}-z_{l}\right|
$$

taken over all collections of points $z_{1}, \ldots, z_{n}$ in the set $E$. Following Fékete, see [21], the quantity $\tau(E)$ is called the transfinite diameter of the set $E$.

Remark 6. Thus, we see that if $C(E)=0$, then $C(f(E))=0$ for an arbitrary mapping $f$ that is continuous by Hölder and, in particular, for quasiconformal mappings on compact sets, see e.g. Theorem II.4.3 in [55].

In order to introduce sets that are measurable with respect to logarithmic capacity, we define, following [18], inner $C_{*}$ and outer $C^{*}$ capacities:

$$
C_{*}(E):=\sup _{F \subseteq E} C(E), \quad C^{*}(E):=\inf _{E \subseteq O} C(O)
$$

where supremum is taken over all compact sets $F \subset \mathbb{C}$ and infimum is taken over all open sets $O \subset \mathbb{C}$. A set $E \subset \mathbb{C}$ is called measurable with respect to the logarithmic capacity if $C^{*}(E)=C_{*}(E)$, and the common value of $C_{*}(E)$ and $C^{*}(E)$ is still denoted by $C(E)$. 
A function $\varphi: E \rightarrow \mathbb{C}$ defined on a bounded set $E \subset \mathbb{C}$ is called measurable with respect to logarithmic capacity if, for all open sets $O \subseteq \mathbb{C}$, the sets $\{z \in E: \varphi(z) \in O\}$ are measurable with respect to logarithmic capacity. It is clear from the definition that the set $\mathrm{E}$ is itself measurable with respect to logarithmic capacity.

Note also that sets of logarithmic capacity zero coincide with sets of the so-called absolute harmonic measure zero introduced by Nevanlinna, see Chapter $\mathrm{V}$ in [61]. Hence a set $E$ is of (Hausdorff) length zero if $C(E)=0$, see Theorem V.6.2 in [61]. However, there exist sets of length zero having a positive logarithmic capacity, see e.g. Theorem IV.5 in [18].

Remark 7. It is known that Borel sets and, in particular, compact and open sets are measurable with respect to logarithmic capacity, see e.g. Lemma I.1 and Theorem III.7 in [18]. Moreover, as it follows from the definition, any set $E \subset \mathbb{C}$ of finite logarithmic capacity can be represented as a union of a sigma-compactum (union of countable collection of compact sets) and a set of logarithmic capacity zero. Thus, the measurability of functions with respect to logarithmic capacity is invariant under Hölder continuous change of variables.

It is also known that the Borel sets and, in particular, compact sets are measurable with respect to all Hausdorff's measures and, in particular, with respect to measure of length, see e.g. theorem $\operatorname{II}(7.4)$ in [67]. Consequently, any set $E \subset \mathbb{C}$ of finite logarithmic capacity is measurable with respect to measure of length. Thus, on such a set any function $\varphi: E \rightarrow \mathbb{C}$ being measurable with respect to logarithmic capacity is also measurable with respect to measure of length on $E$. However, there exist functions that are measurable with respect to measure of length but not measurable with respect to logarithmic capacity, see e.g. Theorem IV.5 in [18].

Dealing with measurable boundary functions $\varphi(\zeta)$ with respect to the logarithmic capacity, we will use the abbreviation q.e. (quasi-everywhere) on a set $E \subset \mathbb{C}$, if a property holds for all $\zeta \in E$ except its subset of zero logarithmic capacity, see [52].

\section{The Dirichlet problem with measurable data in the unit disk for the Poisson equations}

We start with the following analog of the known Luzin theorem on the primitive, see e.g. Theorem VII(2.3) in [67], in terms of logarithmic capacity. 
Proposition 3. [19]. Let $\varphi:[a, b] \rightarrow \mathbb{R}$ be a measurable function with respect to logarithmic capacity. Then there is a continuous function $\Phi:[a, b] \rightarrow \mathbb{R}$ with $\Phi^{\prime}(x)=\varphi(x)$ q.e. on $(a, b)$. Furthermore, $\Phi$ can be chosen with $\Phi(a)=\Phi(b)=0$ and $|\Phi(x)| \leq \varepsilon$ under arbitrary prescribed $\varepsilon>0$ for all $x \in[a, b]$.

As a consequence of Proposition 3, we obtain the following statement.

Proposition 4. Let $\varphi: \partial \mathbb{D} \rightarrow \mathbb{R}$ be a measurable function with respect to logarithmic capacity. Then there is a continuous function $\Phi$ : $\partial \mathbb{D} \rightarrow \mathbb{R}$ such that $\Phi^{\prime}\left(e^{i t}\right)=\varphi\left(e^{i t}\right)$ q.e. on $\mathbb{R}$.

The Poisson-Stieltjes integral

$$
\Lambda_{\Phi}(z):=\frac{1}{2 \pi} \int_{-\pi}^{\pi} P_{r}(\vartheta-t) d \Phi\left(e^{i t}\right), \quad z=r e^{i \vartheta}, r<1, \vartheta \in \mathbb{R}
$$

is well-defined for arbitrary continuous functions $\Phi: \partial \mathbb{D} \rightarrow \mathbb{R}$, see e.g. Section 3 in [66].

Directly by the definition of the Riemann-Stieltjes integral and the Weierstrass type theorem for harmonic functions, see e.g. Theorem I.3.1 in [29], $\Lambda_{\Phi}$ is a harmonic function in the unit disk $\mathbb{D}:=\{z \in \mathbb{C}:|z|<1\}$ because the function $P_{r}(\vartheta-t)$ is the real part of the analytic function

$$
\mathcal{A}_{\zeta}(z):=\frac{\zeta+z}{\zeta-z}, \quad \zeta=e^{i t}, \quad z=r e^{i \vartheta}, r<1, \vartheta \text { and } t \in \mathbb{R} .
$$

Next, by Theorem 1 in [66] we have the following useful conclusion.

Proposition 5. Let $\varphi: \partial \mathbb{D} \rightarrow \mathbb{R}$ be a measurable function with respect to logarithmic capacity and $\Phi: \partial \mathbb{D} \rightarrow \mathbb{R}$ be a continuous function with $\Phi^{\prime}\left(e^{i t}\right)=\varphi\left(e^{i t}\right)$ q.e. on $\mathbb{R}$. Then $\Lambda_{\Phi}$ has the angular limit

$$
\lim _{z \rightarrow \zeta} \Lambda_{\Phi}(z)=\varphi(\zeta) \quad \text { q.e. on } \partial \mathbb{D} \text {. }
$$

Thus, by Lemma 3 and Proposition 5 and the known Poisson formula, see e.g. I.D.2 in [49], we come to the following result on the existence, regularity and representation of solutions for the Dirichlet problem with measurable data to the Poisson equation in the unit disk $\mathbb{D}$. We assume that the charge density $g$ is extended by zero outside of $\mathbb{D}$ in the next theorem.

Theorem 6. Let a function $\varphi: \partial \mathbb{D} \rightarrow \mathbb{R}$ be measurable with respect to the logarithmic capacity and let a continuous function $\Phi$ correspond 
to $\varphi$ by Proposition 4. Suppose that a function $g: \mathbb{D} \rightarrow \mathbb{R}$ is in the class $L^{p}(\mathbb{D})$ for $p>1$. Then the following function in $\mathbb{D}$

$$
U:=N_{g}-\mathcal{P}_{N_{g}^{*}}+\Lambda_{\Phi}, \quad N_{g}^{*}:=\left.N_{g}\right|_{\partial \mathbb{D}},
$$

belongs to the class $W_{\mathrm{loc}}^{2, p}(\mathbb{D})$, satisfies the Poisson equation $\triangle U=g$ a.e. in $\mathbb{D}$ and has the angular limit

$$
\lim _{z \rightarrow \zeta} U(z)=\varphi(\zeta) \quad \text { q.e. on } \partial \mathbb{D} \text {. }
$$

Moreover, $U \in W_{\text {loc }}^{1, q}(\mathbb{D})$ for some $q>2$ and $U$ is locally Hölder continuous. Furthermore, $U \in C_{\text {loc }}^{1, \alpha}(\mathbb{D})$ with $\alpha=(p-2) / p$ if $g \in L^{p}(\mathbb{D})$ for $p>2$.

Remark 8. Note that by the Luzin result, see also Theorem 2 in [66], the statement of Theorem 6 is valid in terms of the length measure as well as the harmonic measure on $\partial \mathbb{D}$.

\section{The Dirichlet problem with measurable data in almost smooth domains}

We say that a Jordan curve $\Gamma$ in $\mathbb{C}$ is almost smooth if $\Gamma$ has a tangent q.e. Here it is said that a straight line $L$ in $\mathbb{C}$ is tangent to $\Gamma$ at a point $z_{0} \in \Gamma$ if

$$
\limsup _{z \rightarrow z_{0}, z \in \Gamma} \frac{\operatorname{dist}(z, L)}{\left|z-z_{0}\right|}=0 .
$$

In particular, $\Gamma$ is almost smooth if $\Gamma$ has a tangent at all its points except a countable set. The nature of such Jordan curves $\Gamma$ is complicated enough because the countable set can be everywhere dense in $\Gamma$.

Given a domain $D$ in $\mathbb{C}, k_{D}\left(z, z_{0}\right)$ denotes the quasihyperbolic distance,

$$
k_{D}\left(z, z_{0}\right):=\inf _{\gamma} \int_{\gamma} \frac{d s}{d(\zeta, \partial D)},
$$

introduced in the paper [24]. Here $d(\zeta, \partial D)$ denotes the Euclidean distance from the point $\zeta \in D$ to $\partial D$ and the infimum is taken over all rectifiable curves $\gamma$ joining the points $z$ and $z_{0}$ in $D$.

Next, it is said that a domain $D$ satisfies the quasihyperbolic boundary condition if

$$
k_{D}\left(z, z_{0}\right) \leq a \ln \frac{d\left(z_{0}, \partial D\right)}{d(z, \partial D)}+b \quad \forall z \in D
$$


for constants $a$ and $b$ and a point $z_{0} \in D$. The latter notion was introduced in [23] but, before it, was first applied in [6].

Remark 9. Given a Jordan domain $D$ in $\mathbb{C}$ with the almost smooth boundary satisfying the quasihyperbolic boundary condition. By the Riemann theorem, see e.g. Theorem II.2.1 in [29], there is a conformal mapping $f: D \rightarrow \mathbb{D}$ that is extended to a homeomorphism $\tilde{f}: \bar{D} \rightarrow \overline{\mathbb{D}}$ by the Caratheodory theorem, see e.g. Theorem II.3.4 in [29]. Moreover, $f_{*}:=\left.\tilde{f}\right|_{\partial D}$, as well as $f_{*}^{-1}$, is Hölder continuous by Corollary to Theorem 1 in [6]. Thus, by Remark 7 a function $\varphi: \partial D \rightarrow \mathbb{R}$ is measurable with respect to logarithmic capacity if and only if the function $\psi:=\varphi \circ f_{*}^{-1}: \partial \mathbb{D} \rightarrow \mathbb{R}$ is so. Set $\Phi:=\Psi \circ f_{*}$ where $\Psi: \partial \mathbb{D} \rightarrow \mathbb{R}$ is a continuous function corresponding to $\psi$ by Proposition 4 .

Proposition 6. Let $D$ be a Jordan domain in $\mathbb{C}$ with the almost smooth boundary satisfying the quasihyperbolic boundary condition. Suppose that $\varphi: \partial D \rightarrow \mathbb{R}$ is measurable with respect to logarithmic capacity and $\Phi: \partial D \rightarrow \mathbb{R}$ is the continuous function corresponding to $\varphi$ by Remark 9. Then the harmonic function $\mathcal{L}_{\Phi}(z):=\Lambda_{\Phi \circ f_{*}^{-1}}(f(z))$ has the angular limit $\varphi$ q.e. on $\partial D$.

Proof. Indeed, by Remark 9 and Proposition 5 there is the angular limit

$$
\lim _{w \rightarrow \xi} \Lambda_{\Psi}(w)=\psi(\xi) \quad \text { q.e. on } \partial \mathbb{D} .
$$

By the Lindelöf theorem, see e.g. Theorem II.C.2 in [49], if $\partial D$ has a tangent at a point $\zeta$, then

$$
\arg [\tilde{f}(\zeta)-\tilde{f}(z)]-\arg [\zeta-z] \rightarrow \text { const } \quad \text { as } z \rightarrow \zeta .
$$

After the change of variables $\xi:=\tilde{f}(\zeta)$ and $w:=\tilde{f}(z)$, we have that

$$
\arg [\xi-w]-\arg \left[\tilde{f}^{-1}(\xi)-\tilde{f}^{-1}(w)\right] \rightarrow \text { const } \quad \text { as } w \rightarrow \xi .
$$

In other words, the conformal images of sectors in $\mathbb{D}$ with a vertex at $\xi$ is asymptotically the same as sectors in $D$ with a vertex at $\zeta$. Thus, nontangential paths in $\mathbb{D}$ are transformed under $\tilde{f}^{-1}$ into nontangential paths in $D$.

Recall that firstly the almost smooth Jordan curve $\partial D$ has a tangent q.e., secondly by Remark 6 the mappings $f_{*}$ and $f_{*}^{-1}$ are Hölder continuous, and thirdly by Remark 7 they transform sets of logarithmic capacity zero into sets of logarithmic capacity zero. Thus, (9.4) implies the desired conclusion. 
Finally, by Lemma 3, Proposition 6 and the Poisson formula, we come to the following result on the existence, regularity and representation of solutions for the Dirichlet problem with measurable data to the Poisson equation in the Jordan domains. We assume here that the charge density $g$ is extended by zero outside of $D$ in the next theorem.

Theorem 7. Let $D$ be a Jordan domain in $\mathbb{C}$ with the almost smooth boundary satisfying the quasihyperbolic boundary condition, a function $\varphi: \partial D \rightarrow \mathbb{R}$ be measurable with respect to logarithmic capacity and let a continuous function $\Phi$ correspond to $\varphi$ by Remark 9. Suppose that a function $g: D \rightarrow \mathbb{R}$ is in the class $L^{p}(D)$ for $p>1$. Then the following function in $D$

$$
U:=N_{g}-\mathcal{D}_{N_{g}^{*}}+\mathcal{L}_{\Phi}, \quad N_{g}^{*}:=\left.N_{g}\right|_{\partial D},
$$

belongs to the class $W_{\mathrm{loc}}^{2, p}(D)$, satisfies the Poisson equation $\triangle U=g$ a.e. in $D$ and has the angular limit

$$
\lim _{z \rightarrow \zeta} U(z)=\varphi(\zeta) \quad \text { q.e. on } \partial D .
$$

Moreover, $U \in W_{\text {loc }}^{1, q}(D)$ for some $q>2$ and $U$ is locally Hölder continuous. Furthermore, $U \in C_{\text {loc }}^{1, \alpha}(D)$ with $\alpha=(p-2) / p$ if $g \in L^{p}(D)$ for $p>2$.

Remark 10. Note that by the Luzin result, see also Theorem 3 in [66], the statement of Theorems 7 is valid in terms of the length measure on rectifiable $\partial D$. Indeed, by the Riesz theorem length $f_{*}^{-1}(E)=0$ whenever $E \subset \partial \mathbb{D}$ with $|E|=0$, see e.g. Theorem II.C.1 and Theorems II.D.2 in [49]. Conversely, by the Lavrentiev theorem $\left|f_{*}(\mathcal{E})\right|=0$ whenever $\mathcal{E} \subset \partial D$ and length $\mathcal{E}=0$, see [53], see also the point III.1.5 in [63].

However, by the well-known Ahlfors-Beurling example, see [2], the sets of length zero as well as of harmonic measure zero are not invariant with respect to quasiconformal changes of variables. The latter circumstance does not make it is possible to apply the result in the future for the extension of the statement to generalizations of the Poisson equation in anisotropic and inhomogeneous media. Hence we prefer to use logarithmic capacity.

\section{Acknowledgments}

This work was partially supported by grant of Ministry of Education and Science of Ukraine, project number is 0119U100421. 


\section{References}

[1] L. V. Ahlfors, Lectures on quasiconformal mappings, Van Nostrand Mathematical Studies, 10 (1966), Toronto, Ont.-New York-London, Van Nostrand Co., Inc.; transl. as Lektsii po kvazikonformnym otobrazheniyam, Mir, Moscow, 1969.

[2] L. Ahlfors, A. Beurling, The boundary correspondence under quasiconformal mappings // Acta Math., 96 (1956), 125-142.

[3] R. Aris, The Mathematical Theory of Diffusion and Reaction in Permeable Catalysts, V, I-II, Oxford, Clarendon Press, 1975.

[4] K. Astala, T. Iwaniec, G. Martin, Elliptic partial differential equations and quasiconformal mappings in the plane, Princeton Mathematical Series 48, Princeton, Princeton University Press, NJ, 2009.

[5] G. I. Barenblatt, Ja. B. Zel'dovic, V. B. Librovich, G. M. Mahviladze, Matematicheskaya teoriya goreniya i vzryva, Moscow, Nauka, 1980, (in Russian); transl. The mathematical theory of combustion and explosions, New York, Consult. Bureau, 1985.

[6] J. Becker, Ch. Pommerenke, Hölder continuity of conformal mappings and nonquasiconformal Jordan curves // Comment. Math. Helv., 57 (1982), No. 2, 221-225.

[7] E. Beltrami, Saggio di interpretazione della geometria non euclidea, Giornale di Mathematica, 6 (1867).

[8] L. Bers, Mathematical aspects of subsonic and transonic gas dynamics // Surveys in Applied Math., 3, John Wiley, New York; Chapman and Hall, London, 1958.

[9] L. Bers, L. Nirenberg, On a representation theorem for linear elliptic systems with discontinuous coefficients and its applications // Convegno Internazionale sulle Equazioni Lineari alle Derivate Parziali, Trieste, 1954, 111-140, Edizioni Cremonese, Rome, 1955, 111-140.

[10] L. Bieberbach, $\triangle u=e^{u}$ und die automorphen Funktionen // Math. Ann., 7 (1916), No. 7, 173-212.

[11] B. V. Bojarski, Homeomorphic solutions of Beltrami systems // Dokl. Akad. Nauk SSSR (N.S.), 102 (1955), 661-664.

[12] B. V. Bojarski, Generalized solutions of a system of differential equations of the first order and elliptic type with discontinuous coefficients // Mat. Sb. (N.S.), 43(85) (1957), 451-503; transl. in Report Dept. Math. Stat., 118 (2009).

[13] B. Bojarski, V. Gutlyanskii, O. Martio, V. Ryazanov, Infinitesimal geometry of quasiconformal and bi-lipschitz mappings in the plane, EMS Tracts in Mathematics, 19, European Mathematical Society (EMS), Zurich, 2013.

[14] B. Bojarski, V. Gutlyanskii, V. Ryazanov, On the Beltrami equations with two characteristics // Complex Var. Elliptic Equ., 54 (2009), No. 10, 935-950.

[15] B. Bojarski, T. Iwaniec, Analytical foundations of the theory of quasiconformal mappings in $\mathbb{R}^{n} / /$ Ann. Acad. Sci. Fenn. Ser. A I Math., 8 (1983), No. 2, $257-324$.

[16] J. I. Diaz, Nonlinear partial differential equations and free boundaries. V. I. // Elliptic equations. Research Notes in Mathematics, 106 (1985).

[17] N. Dunford, J. T. Schwartz, Linear Operators. I. General Theory, Pure and Applied Mathematics 7 (1958). New York, London, Interscience Publishers. 
[18] L. Carleson, Selected Problems on Exceptional Sets, Princeton etc., Van Nostrand Co., Inc, 1971.

[19] A. S. Efimushkin, V. I. Ryazanov, On the Riemann-Hilbert problem for the Beltrami equations in quasidisks //. Ukr. Mat. Visn., 12 (2015), No. 2, 190-209; transl. in J. Math. Sci. (N.Y.), 211 (2015), No. 5, 646-659.

[20] H. Federer, Geometric Measure Theory, Berlin: Springer-Verlag, 1969; transl. in Geometricheskaya teoriya mery, Moscow, Nauka, 1987.

[21] M. Fékete, Über die Verteilung der Wurzeln bei gewissen algebraischen Gleichungen mit ganzzahligen Koeffizienten // Math. Z., 17 (1923), 228-249.

[22] J. B. Garnett, D. E. Marshall, Harmonic Measure, Cambridge, Cambridge Univ. Press, 2005.

[23] F. W. Gehring, O. Martio, Lipschitz classes and quasiconformal mappings // Ann. Acad. Sci. Fenn. Ser. A I Math., 10 (1985), 203-219.

[24] F. W. Gehring, B. P. Palka, Quasiconformally homogeneous domains // J. Analyse Math., 30 (1976), 172-199.

[25] M. Giaquinta, L. Martinazzi, An introduction to the regularity theory for elliptic systems, harmonic maps and minimal graphs, Second edition. Appunti. Scuola Normale Superiore di Pisa (Nuova Serie) [Lecture Notes. Scuola Normale Superiore di Pisa (New Series)], 11. Edizioni della Normale, Pisa, 2012.

[26] D. Gilbarg, N. Trudinger, Elliptic partial differential equations of second order, Grundlehren der Mathematischen Wissenschaften, 224, Berlin, SpringerVerlag, 1983; Ellipticheskie differentsial'nye uravneniya s chastnymi proizvodnymi vtorogo poryadka, Moscow, Nauka, 1989.

[27] V. Gol'dshtein, V. Pchelintsev, A. Ukhlov, Composition operators on Sobolev spaces and Eigenvalues of Divergent Elliptic Operators, arXiv:1903.11301v1 [math.AP], 27 Mar 2019, 1-20.

[28] V. Gol'dshtein, A. Ukhlov, About homeomorphisms that induce composition operators on Sobolev spaces // Complex Var. Elliptic Equ., 55 (2010), No. 8-10, $833-845$.

[29] G. M. Goluzin, Geometric theory of functions of a complex variable, Transl. of Math. Monographs 26, Providence, R.I.: American Mathematical Society, 1969.

[30] A. K. Gushchin, On the Dirichlet problem for a second-order elliptic equation // Math. USSR-Sb., 65 (1990), No. 1, 19-66; translated from Math. Sb., 137(179) (1988), No. 1(9), 19-64.

[31] V. Ya. Gutlyanskiǔ, O. Martio, Conformality of a quasiconformal mapping at a point // J. Anal. Math. 91 (2003), 179-192.

[32] V. Gutlyanskiǔ, O. Martio, Rotation estimates and spirals // Conform. Geom. Dyn., 5 (2001), 6-20.

[33] V. Ya. Gutlyanskii, O. V. Nesmelova, V. I. Ryazanov, On a model semilinear elliptic equation in the plane // Ukr. Mat. Visn., 13 (2016), No. 1, 91-105; translated in J. Math. Sci. (N.Y.), 220 (2017), No. 5, 603-614.

[34] V. Ya. Gutlyanskii, O. V. Nesmelova, V. I. Ryazanov, Semilinear equations in a plane and quasiconformal mappings // Dopov. Nats. Akad. Nauk Ukr. Mat. Prirodozn. Tekh. Nauki, (2017), No. 1, 10-16.

[35] V. Ya. Gutlyanskii, O. V. Nesmelova, V. I. Ryazanov, On the Dirichlet problem for quasilinear Poisson equations // Proceedings of IAMM of NAS of Ukraine, 31 (2017), 28-37. 
[36] V. Ya. Gutlyanskii, O. V. Nesmelova, V. I. Ryazanov, On quasiconformal maps and semi-linear equations in the plane // Ukr. Mat. Visn., 14 (2017), No. 2, 161-191; translated in J. Math. Sci. 229 (2018), No. 1, 7-29.

[37] V. Ya. Gutlyanskii, O. V. Nesmelova, V. I. Ryazanov, Semilinear equations in the plane with measurable data // Dopov. Nats. Akad. Nauk Ukr. Mat. Prirodozn. Tekh. Nauki, (2018), No. 2, 12-18.

[38] V. Ya. Gutlyanskii, O. V. Nesmelova, V. I. Ryazanov, On blow-up solutions and dead zones in semilinear equations // Dopov. Nats. Akad. Nauk Ukr. Mat. Prirodozn. Tekh. Nauki, (2018), No. 4, 9-15.

[39] V. Ya. Gutlyanskii, O. V. Nesmelova, V. I. Ryazanov, On the regularity of solutions of quasilinear Poisson equations // Dopov. Nats. Akad. Nauk Ukr. Mat. Prirodozn. Tekh. Nauki, (2018), No. 10, 9-17.

[40] V. Ya. Gutlyanskii, V. I. Ryazanov, Quasiconformal mappings in the theory of semi-linear equations // Lobachevskii J. Math., 39 (2018), No. 9, 1343-1352.

[41] V. Gutlyanskii, V. Ryazanov, U. Srebro, E. Yakubov, On recent advances in the Beltrami equations // Journal of Math. Sci. (N.Y.), 175 (2011), No. 4, 413-449 ; translation from Ukr. Mat. Visn., 7 (2010), No. 4, 467-515.

[42] V. Gutlyanskii, V. Ryazanov, U. Srebro, E. Yakubov, The Beltrami equation. A geometric approach, Developments in Mathematics, 26, New York, Springer, 2012 .

[43] V. Gutlyanskii, V. Ryazanov, E. Yakubov, The Beltrami equations and prime ends // Journal of Math. Sci. (N.Y.) 210 (2015), No. 1, 22-51; translation from Ukr. Mat. Visn., 12 (2015), No. 1, 27-66.

[44] V. Gutlyanskii, V. Ryazanov, A. Yefimushkin, On the boundary value problems for quasiconformal functions in the plane // Ukr. Mat. Visn. 12 (2015), No. 3, 363-389; transl. in J. Math. Sci. (N.Y.) 214 (2016), No. 2, 200-219.

[45] L. Hörmander, Notions of convexity, Progress in Mathematics, 127, Boston, Birkhäuser Boston Inc, 1994.

[46] L. Hörmander, The analysis of linear partial differential operators. V. I. Distribution theory and Fourier analysis, Grundlehren der Mathematischen Wissenschaften 256, Springer-Verlag, Berlin, 1983; transl. as Analiz lineinykh differentsial'nykh operatorov s chastnymi proizvodnymi, T. 1, Moscow, Mir, 1986.

[47] W. Hurewicz, H. Wallman, Dimension Theory, Princeton, Princeton Univ. Press, 1948.

[48] A. A. Ignat'ev, V. I. Ryazanov, Finite mean oscillation in mapping theory // Ukr. Mat. Visn., 2 (2005), No. 3, 395-417; transl. in Ukr. Math. Bull. 2 (2005), No. 3, 403-424.

[49] P. Koosis, Introduction to $H^{p}$ spaces, Cambridge Tracts in Mathematics, 115, Cambridge, Cambridge Univ. Press, 1998.

[50] K. Kuratowski, Topology. Vol. II, New York-London, Academic Press, 1968.

[51] O. A. Ladyzhenskaya, N. N. Ural'tseva, Linear and quasilinear elliptic equations, Academic Press, New York-London, 1968; transl. from Lineinye $i$ kvazilineinye uravneniya ellipticheskogo tipa, Nauka, Moscow 1964.

[52] N. S.Landkof, Foundations of modern potential theory, Moscow, Nauka, 1966; Grundlehren der mathematischen Wissenschaften, 180, New York-Heidelberg, Springer-Verlag, 1972. 
[53] M. Lavrentiev, On some boundary problems in the theory of univalent functions // Mat. Sbornik N.S., 1(43) (1936), No. 6, 815-846 (in Russian).

[54] M. Lavrentiev, Sur une critére differentiel des transformations homéomorphes des domains á trois dimensions // Dokl. Acad. Nauk. SSSR, 20 (1938), 241-242.

[55] O. Lehto, K. J. Virtanen, Quasiconformal mappings in the plane, Berlin, Heidelberg, Springer-Verlag, 1973.

[56] J. Leray, Ju. Schauder, Topologie et equations fonctionnelles // Ann. Sci. Ecole Norm. Sup., 51 (1934), No. 3, 45-78 (in French); transl. Topology and functional equations // Uspehi Matem. Nauk (N.S.). 1 (1946), No. 3-4 (13-14), 71-95.

[57] O. Martio, V. Ryazanov, U. Srebro, E. Yakubov, Moduli in modern mapping theory, Springer Monographs in Mathematics, New York, Springer, 2009.

[58] M. Marcus, L. Veron, Nonlinear second order elliptic equations involving measures, De Gruyter Series in Nonlinear Analysis and Applications, 21, De Gruyter, Berlin, 2014.

[59] S. G. Mihlin, Linear partial differential equations, Moscow, Izdat. Vysh. Shkola, 1977 (in Russian).

[60] C. B. Morrey, On the solutions of quasi-linear elliptic partial differential quations // Trans. Amer. Math. Soc., 43 (1938), 126-166.

[61] R. Nevanlinna, Eindeutige analytische Funktionen // Michigan, Ann Arbor, 1944.

[62] S. I. Pokhozhaev, On an equation of combustion theory // Mat. Zametki, 88 (2010), No. 1, 53-62; translated in Math. Notes, 88 (2010), No. 1-2, 48-56.

[63] I. I. Priwalow, Randeigenschaften analytischer Funktionen, Hochschulbücher für Mathematik 25, Berlin, Deutscher Verlag der Wissenschaften, 1956.

[64] T. Ransford, Potential theory in the complex plane // London Mathematical Society Student Texts 28, Cambridge, Cambridge University Press, 1995.

[65] W. Rudin, Real and complex analysis, New York, McGraw-Hill Book Co, 1987.

[66] V. Ryazanov, The Stieltjes integrals in the theory of harmonic functions // Zap. Nauchn. Sem. S.-Peterburg. Otdel. Mat. Inst. Steklova (POMI) 467, Issledovaniya po Lineinym Operatoram i Teorii Funktsii 46 (2018), 151-168, ftp.pdmi.ras.ru/pub/publicat/znsl/v467/p151.pdf.

[67] S. Saks, Theory of the integral, Warsaw, 1937; Dover Publications Inc., New York, 1964; transl. as Teoria integrala, Inostran. Lit., Moscow, 1949.

[68] J. Serrin, Local behaviour of solutions of quasilinear equations // Acta Math., 111 (1964), 247-302.

[69] S. L. Sobolev, Some applications of functional analysis in mathematical physics // Transl. Math. Mon. 90, AMS, Providence, RI, 1991; from Nekotorye primeneniya funkcional'nogo analiza $v$ matematiceskoi fizike, Nauka, Moscow, 1988.

[70] A. Ukhlov, Mappings generating the embeddings of Sobolev spaces // Sib. Mat. Zh., 34 (1993), No. 1, 165-171.

[71] I. N. Vekua, Generalized analytic functions, Pergamon Press, London-ParisFrankfurt; Addison-Wesley Publishing Co., Inc., Reading, Mass., 1962; transl. from Obobshchennye analiticheskie funktsii, Fiz.-Mat. Lit., Moscow, 1959.

[72] I. N. Vekua, Some properties of solutions of Gauss equation // Trudy Mat. Inst. Steklov, 64 (1961), 5-8. 
[73] L. Veron, Local and global aspects of quasilinear degenerate elliptic equations, Quasilinear elliptic singular problems, World Scientific Publishing Co. Pte. Ltd., Hackensack, NJ., 2017.

[74] S. K. Vodopyanov, A. Ukhlov, Sobolev spaces and (P; Q)-quasiconformal mappings of Carnot groups // Sib. Mat. Zh., 39 (1998), No. 4, 665-682.

\section{CONTACT INFORMATION}

Vladimir

Gutlyanskiı̌

Olga Nesmelova

Vladimir Ryazanov
Institute of Applied Mathematics

and Mechanics of the NAS of Ukraine, Slavyansk, Ukraine

E-Mail: vgutlyanskii@gmail.com

Institute of Applied Mathematics

and Mechanics of the NAS of Ukraine,

Slavyansk, Ukraine

E-Mail: star-o@ukr.net

Institute of Applied Mathematics

and Mechanics of the NAS of Ukraine, Slavyansk, Ukraine,

Bogdan Khmelnytsky National

University of Cherkasy,

Cherkasy, Ukraine

E-Mail: Ryazanov@nas.gov.ua,

v1.ryazanov1@gmail.com 